\title{
Full-dimensional treatment of short-time vibronic dynamics in a molecular high-order-harmonic-generation process in methane
}

\author{
Serguei Patchkovskii ${ }^{*}$ \\ Max Born Institute, Max-Born-Straße 2A, 12489 Berlin, Germany \\ Michael S. Schuurman \\ Department of Chemistry and Biomolecular Sciences, University of Ottawa, D'Iorio Hall, 10 Marie Curie, Ottawa, Ontario, Canada K1N 6N5 \\ and National Research Council of Canada, 100 Sussex Drive, Ottawa, Ontario, Canada K1A OA6
}

(Received 13 September 2017; published 9 November 2017)

\begin{abstract}
We present derivation and implementation of the multiconfigurational strong-field approximation with Gaussian nuclear wave packets (MC-SFA-GWP) - a version of the molecular strong-field approximation which treats all electronic and nuclear degrees of freedom, including their correlations, quantum mechanically. The technique allows realistic simulation of high-order-harmonic emission in polyatomic molecules without invoking reduced-dimensionality models for the nuclear motion or the electronic structure. We use MC-SFA-GWP to model isotope effects in high-order-harmonic-generation (HHG) spectroscopy of methane. The HHG emission in this molecule transiently involves the strongly vibronically coupled ${ }^{2} F_{2}$ electronic state of the $\mathrm{CH}_{4}{ }^{+}$cation. We show that the isotopic HHG ratio in methane contains signatures of (a) field-free vibronic dynamics at the conical intersection (CI); (b) resonant features in the recombination cross sections; (c) laser-driven bound-state dynamics; as well as (d) the well-known short-time Gaussian decay of the emission. We assign the intrinsic vibronic feature (a) to a relatively long-lived $(\geqslant 4 \mathrm{fs})$ vibronic wave packet of the singly excited $v_{4}\left(t_{2}\right)$ and $v_{2}(e)$ vibrational modes, strongly coupled to the components of the ${ }^{2} F_{2}$ electronic state. We demonstrate that these physical effects differ in their dependence on the wavelength, intensity, and duration of the driving pulse, allowing them to be disentangled. We thus show that HHG spectroscopy provides a versatile tool for exploring both conical intersections and resonant features in photorecombination matrix elements in the regime not easily accessible with other techniques.
\end{abstract}

DOI: 10.1103/PhysRevA.96.053405

\section{INTRODUCTION}

One of the elusive targets being pursued by the rapidly developing research area of strong-field and attosecond science is following the electronic and nuclear motion in atoms and molecules on their natural, atto- and femtosecond time scales [1-6]. High-order-harmonic spectroscopy (HHS) [7], based on the celebrated high-order-harmonic-generation (HHG) process $[8,9]$, is one of the most powerful and versatile tools in the arsenal of attosecond science. Among other examples $[10,11]$, it has been successfully employed to image the "molecular orbitals" [3,12-14] and resolve multiple final states in strong-field ionization [15,16]; follow evolution of bound electronic [17-25], rotational [26-28], and nuclear [16,29] wave packets; monitor electron correlation in atoms [30]; measure molecular chirality [31,32]; resolve the time tunneling electron emerges from underneath the barrier [33]; probe strongly driven electrons in the continuum [18,34-36]; and control attosecond emission from molecules [37,38].

HHG is a highly nonlinear process $[9,39]$, which often makes the interpretation of experimental results complex and controversial. Similar to conventional spectroscopies,

\footnotetext{
*Serguei.Patchkovskii@mbi-berlin.de

Published by the American Physical Society under the terms of the Creative Commons Attribution 4.0 International license. Further distribution of this work must maintain attribution to the author(s) and the published article's title, journal citation, and DOI.
}

measurement of the isotope effects and quantum-path interferences in HHG has emerged as one of the most helpful techniques in the detailed analysis of high-order-harmonic spectra $[15,40-49]$. In the form of the PACER (probing attosecond dynamics by chirp-encoded recollision [50]) experiment, isotope-dependent HHS promises direct access to the electronic and nuclear dynamics on a few- and subfemtosecond time scale, including measurement of the phases of the nuclear wave packets $[44,45]$.

For the electronically simple cases, where the BornOppenheimer (BO) variable separation applies, the theory underlying PACER experiments is well understood [40,51]. The relevant theory object, the short-time nuclear autocorrelation function [40], is readily available for most small molecules [52]. At the same time, the $\mathrm{BO}$ assumptions already fail for one of the first molecules examined with PACER spectroscopy. In methane, $\mathrm{CH}_{4}$, the key dynamics occur in the vicinity of a symmetry-required triple electronic surface intersection in a transiently prepared $\mathrm{CH}_{4}{ }^{+}$cation. In larger, more electronically complex systems, which are beginning to be explored with HHS [53-60] (see Ref. [61] for a review), conical intersections and nonseparable vibronic dynamics are expected to become ubiquitous $[62,63]$.

The effects of the non-Born-Oppenheimer dynamics and isotope effects in HHG have been studied for more than 20 years [64]. Nonetheless, most of the non-BO numerical studies of HHG still concern the dynamics of simple, oneand two-electron diatomics $\left(\mathrm{H}_{2}\right.$ [65-68], $\mathrm{H}_{2}{ }^{+}$[64,66,68-80], $\mathrm{HeH}^{2+}[81]$ ), usually with the additional approximations of the reduced dimensionality $[66-74,77,80,81]$ or special field 
polarizations $[64,75,76,78,79]$. Some attempts at extending the theory of nuclear dynamics in HHG to more general non-BO systems have been reported in the literature [65,82-97]. Nonetheless, no fully satisfactory solution to this problem, which requires simultaneous consideration of nonperturbative continuum electronic dynamics, vibronic dynamics, and their correlations, has become available so far.

The goal of this work is to extend the strong-field approximation (SFA) [39,98,99], a nonperturbative theory underlying much of attosecond science $[2,100]$, to account for the short-time vibronic dynamics. The rest of this work is structured as follows. Section II derives the working expressions for the multiconfigurational strong-field approximation with Gaussian nuclear wave packets (MC-SFA-GWP). Section III gives technical details for the MC-SFA-GWP calculations of HHG emission in methane and deuterated methane, including the details of the electronic structure calculations, the diabatization procedure, and integration of the MC-SFA-GWP equations. Section IV presents the results of the numerical calculations. Specifically, Sec. IV A discusses vibronic nuclear autocorrelation function in methane and analyzes its features in terms of the wave packet composition and dynamics. Section IV B presents calculated HHG spectra for three wavelengths $(800,1600$, and $2400 \mathrm{~nm})$ and two

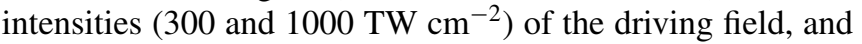
dissects the physical origin of the observed spectral features. Section IV C analyzes calculated PACER signals, compares them to the available experimental data, and makes predictions for the previously unexplored range of the experimental parameters. Section V summarizes the results and offers an outlook for future developments. Finally, some of the technical aspects of the MC-SFA-GWP derivation and implementation are relegated to Appendixes A-C.

\section{THEORY}

Our aim is to model high-order-harmonic emission due to the short-time, coupled electronic and nuclear dynamics of a molecular system under the influence of an intense, longwavelength (near- to mid-IR) laser field. It is convenient to write the total Hamiltonian $\hat{H}_{\mathrm{T}}$ as a sum of three terms, all of which may be time dependent [101-103]:

$$
\hat{H}_{\mathrm{T}}(t)=\hat{P}_{0} \hat{H}_{0}(t) \hat{P}_{0}+\hat{P}_{1} \hat{H}_{1}(t) \hat{P}_{1}+\hat{P}_{1} \hat{V}_{\mathrm{L}}(t) \hat{P}_{0},
$$

where $\hat{P}_{0}$ and $\hat{P}_{1}$ are respectively projectors onto the neutral and singly ionized spaces. The contribution $\hat{H}_{0}(t)$ acts within the subspace of the neutral states. The term $\hat{H}_{1}(t)$ acts within the subspace of singly ionized states, while the interaction Hamiltonian $\hat{V}_{\mathrm{L}}$ describes laser-induced transitions between the two spaces. In the dipole approximation and length gauge,

$$
\hat{V}_{\mathrm{L}}(t)=e \vec{F}(t) \cdot \sum_{i}^{N} \vec{r}_{i},
$$

where $\vec{r}_{i}$ are coordinates of the $i$ th electron and $\vec{F}(t)$ is the laser electric field, which is is assumed to be slowly varying. $N$ is the total number of electrons in the system. In Eq. (1), we have already neglected the possibility of multiple ionization, as well as the stimulated recombination process.
A formally exact solution of the time-dependent Schrödinger equation with Hamiltonian (1) is given by $[99,102,103]$

$$
\begin{aligned}
\Psi(\vec{r}, \vec{q}, t)= & \hat{U}_{0}\left(t, t_{0}\right) \Psi\left(\vec{r}, \vec{q}, t_{0}\right) \\
& -\frac{i}{\hbar} \int_{t_{0}}^{t} d t_{1} \hat{U}_{\mathrm{T}}\left(t, t_{1}\right) \hat{V}_{\mathrm{L}}\left(t_{1}\right) \hat{U}_{0}\left(t_{1}, t_{0}\right) \Psi\left(\vec{r}, \vec{q}, t_{0}\right) .
\end{aligned}
$$

The propagators $\hat{U}_{0}, \hat{U}_{1}$, and $\hat{U}_{\mathrm{T}}$ are

$$
\hat{U}_{\mathrm{X}}\left(t_{j}, t_{i}\right)=\exp \left(-\frac{i}{\hbar} \int_{t_{i}}^{t_{j}} d t^{\prime} \hat{H}_{\mathrm{X}}\left(t^{\prime}\right)\right),
$$

where $X=0,1$, T. In Eq. (3), $\vec{r}$ and $\vec{q}$ are respectively electronic and nuclear coordinates. We assume that the initial wave function at time $t_{0}\left[\Psi\left(\vec{r}, \vec{q}, t_{0}\right)\right]$ corresponds to the neutral species.

Classically, the high-order-harmonic intensity is determined by the power spectrum of the time-dependent dipole $\vec{d}(t)$. Neglecting contributions arising entirely within the neutral and cation manifolds, the radiating dipole is given by

$$
\begin{aligned}
\vec{d}(t)= & -\frac{i}{\hbar} \int d \vec{r} \int d \vec{q} \int_{t_{0}}^{t} d t_{1}\left[\hat{U}_{0}\left(t, t_{0}\right) \Psi\left(\vec{r}, \vec{q}, t_{0}\right)\right]^{*} \\
& \times \hat{V}_{\mathrm{D}} \hat{U}_{\mathrm{T}}\left(t, t_{1}\right) \hat{V}_{\mathrm{L}}\left(t_{1}\right) \hat{U}_{0}\left(t_{1}, t_{0}\right) \Psi\left(\vec{r}, \vec{q}, t_{0}\right)+\text { c.c. },
\end{aligned}
$$

where the recombination dipole operator is

$$
\hat{V}_{\mathrm{D}}=e \sum_{i}^{N} \vec{r}_{i}
$$

with the sum running over coordinates of all electrons.

Equation (5) can be brought into a computationally tractable form by introducing an identity operator $\hat{\mathbf{1}}$, given by a sum of identity operators within the neutral, singly ionized, etc., spaces:

$$
\begin{gathered}
\hat{\mathbf{1}}=\hat{\mathbf{1}}_{0}+\hat{\mathbf{1}}_{1}+\cdots \\
\hat{\mathbf{1}}_{0}=\sum_{a \mathbf{n}}\left|\Phi_{a}\right\rangle\left\langle\Phi_{a}|| \mathbf{n}\right\rangle\langle\mathbf{n}|, \\
\hat{\mathbf{1}}_{1}=\sum_{b \mathbf{m}}|\mathbf{m}\rangle\left\langle\mathbf { m } \left|\int d \vec{k}\left[\hat{A}_{1}\left|X_{b}\right\rangle\left|\vec{k}_{b}\right\rangle\right]\left[\hat{A}_{1}\left\langle X_{b}\right|\left\langle\vec{k}_{b}\right|\right] .\right.\right.
\end{gathered}
$$

The identity operator within the neutral space $\left(\hat{\mathbf{1}}_{0}\right)$ is defined in terms of the neutral electronic states $\left|\Phi_{a}\right\rangle$ (which depend on $\vec{r}$ and parametrically on $\vec{q}$ ) and harmonic nuclear vibrational states $|\mathbf{n}\rangle$ (which depend only on $\vec{q}$ ). Quantity $\mathbf{n}$ is a vector of non-negative integers, with each element $\mathbf{n}_{k}$ defining the excitation level of the $k$ th normal mode of a reference potential $v_{\text {ref }}(\vec{q})$, which is chosen for computational convenience $[52,90]$.

The identity operator within the singly ionized space $\left(\hat{\mathbf{1}}_{1}\right)$ contains bound states of the $(N-1)$-electron ion $\left|X_{b}\right\rangle$ and the corresponding one-electron scattering states $\left|\vec{k}_{b}\right\rangle$ with the asymptotic momentum $\vec{k}_{b}$. Functions $\left|\vec{k}_{b}\right\rangle$ depend on the coordinate of the $N$ th electron $\vec{r}_{N}$. Formally, the sum over $b$ in Eq. (9) does not include autoionizing states $\left|X_{b}\right\rangle$, which belong to the doubly ionized continuum. Such states are unlikely to be populated by strong-field ionization, but may 
become important due to electron-correlation effects in the recombination step [30,104,105]. If the lifetime of such an autoionizing state is long compared to the laser cycle, its effects can still be accounted for, by including the state in Eq. (9).

Both $\left|X_{b}\right\rangle$ and $\left|\vec{k}_{b}\right\rangle$ depend parametrically on $\vec{q}$. Operator $\hat{A}_{1}$ antisymmetrizes the product wave function $\left|X_{b}\right\rangle\left|\vec{k}_{b}\right\rangle$ with respect to the coordinates of the continuum electron $[106,107]$,

$$
\hat{A}_{1}=\frac{1}{\sqrt{N}}\left[\hat{I}-\sum_{i=1}^{N-1} \hat{P}_{i N}\right]
$$

where $\hat{I}$ is the $N$-electron identity operator, and operator $\hat{P}_{i N}$ permutes coordinates of the $i$ th and $N$ th electrons. Similar to the neutral-space identity $\hat{\mathbf{1}}_{0}$, the nuclear wave function in $\hat{\mathbf{1}}_{1}$ is expanded in terms of the harmonic vibrational states $|\mathbf{m}\rangle$ of the reference potential $v_{\text {ref }}(\vec{q})$. Because we are not interested in processes involving multiple ionization, the sum in Eq. (7) is truncated after $\hat{\mathbf{1}}_{1}$.

Operator $\hat{\mathbf{1}}$ as well as the individual $\hat{\mathbf{1}}_{i}$ operators are idempotent. Identity operators within different subspaces are assumed to commute:

$$
\left[\hat{\mathbf{1}}_{0}, \hat{\mathbf{1}}_{1}\right]=0 .
$$

This condition is equivalent to the strong orthogonality assumption for all $\left|\vec{k}_{b}\right\rangle$ :

$$
\begin{gathered}
\left\langle\phi_{b a}^{\mathrm{D}} \mid \vec{k}_{b}\right\rangle=0, \\
\left|\phi_{b a}^{\mathrm{D}}\right\rangle=\sqrt{N}\left\langle X_{b} \mid \Phi_{a}\right\rangle,
\end{gathered}
$$

where $\left|\phi_{b a}^{\mathrm{D}}\right\rangle$ is the Dyson orbital for the ionization of the neutral state $\left|\Phi_{a}\right\rangle$, forming cation $\left|X_{b}\right\rangle$.

We now insert operator $\hat{\mathbf{1}}$ of Eq. (7) to the left and to the right of each propagator and interaction operator in Eq. (5). Following the usual SFA assumptions [99,102,103], we also replace $\hat{U}_{\mathrm{T}}\left(t, t_{1}\right)$ by a product of the $(N-1)$-electron ion propagator $\hat{U}_{\mathrm{I}}\left(t, t_{1}\right)$ and the Volkov propagator $\hat{U}_{\mathrm{V}}\left(t, t_{1}\right)$ :

$$
\begin{gathered}
\hat{U}_{\mathrm{T}}\left(t, t_{1}\right) \approx \hat{U}_{\mathrm{I}}\left(t, t_{1}\right) \hat{U}_{\mathrm{V}}\left(t, t_{1}\right), \\
\hat{U}_{\mathrm{V}}\left(t, t_{1}\right)|\vec{k}\rangle \\
=\left|\vec{k}-\frac{e}{\hbar} \vec{A}\left(t_{1}\right)+\frac{e}{\hbar} \vec{A}(t)\right\rangle \\
\times \exp \left(\frac{-i \hbar}{2 m} \int_{t_{1}}^{t} d t^{\prime}\left(\vec{k}-\frac{e}{\hbar} \vec{A}\left(t_{1}\right)+\frac{e}{\hbar} \vec{A}\left(t^{\prime}\right)\right)^{2}\right), \\
\langle\vec{r} \mid \vec{k}\rangle=\frac{1}{(2 \pi)^{3 / 2}} e^{i \vec{k} \cdot \vec{r}},
\end{gathered}
$$

where $\vec{A}(t)$ is the vector potential of the laser field, and Volkov states $|\vec{k}\rangle$ are taken in the length gauge. The explicit coordinate representation of $|\vec{k}\rangle$ normalized to $\delta\left(\vec{k}-\vec{k}^{\prime}\right)$ is given by Eq. (16). In the simplest form of the SFA used presently, the continuum state $\left|\vec{k}_{b}\right\rangle$ does not depend on the nature of the binding potential. For simplicity, we therefore omit the subscript $b$ in $\left|\vec{k}_{b}\right\rangle$ from now on. (The dependence of $|\vec{k}\rangle$ on the binding potential can of course be reintroduced if necessary $[100,102,103]$, without materially affecting the overall form of the MC-SFA-GWP working expressions.) Finally, propagator
$\hat{U}_{\mathrm{I}}$ is defined similar to Eq. (4), with the $(N-1)$-electron vibronic Hamiltonian given by $\hat{H}_{\mathrm{I}}$.

Neglecting contributions due to correlation-driven inelastic electron scattering in the continuum [106,107], we obtain

$$
\begin{aligned}
& \vec{d}(t)=-\frac{i}{\hbar} \int_{t_{0}}^{t} d t_{1} \int d \vec{k} \sum_{a^{\prime \prime} \mathbf{n}^{\prime \prime}} \sum_{b^{\prime} \mathbf{m}^{\prime}} \sum_{b \mathbf{m}} \sum_{a^{\prime \prime \prime} \mathbf{n}^{\prime \prime \prime}} C_{a^{\prime \prime} \mathbf{n}^{\prime \prime}}^{*}(t) \\
& \times \vec{R}_{b^{\prime} \mathbf{m}^{\prime} a^{\prime \prime} \mathbf{n}^{\prime \prime}}\left(\vec{k}-\frac{e}{\hbar} \vec{A}\left(t_{1}\right)+\frac{e}{\hbar} \vec{A}(t)\right) D_{b^{\prime} \mathbf{m}^{\prime} b \mathbf{m}}\left(t, t_{1}\right) \\
& \times \vec{F}\left(t_{1}\right) \cdot \vec{R}_{b \mathbf{m} a^{\prime \prime \prime} \mathbf{n}^{\prime \prime \prime}}(\vec{k}) C_{a^{\prime \prime \prime} \mathbf{n}^{\prime \prime \prime}}\left(t_{1}\right) e^{-i \phi_{d}\left(\vec{k}, t, t_{1}\right)}+\text { c.c. }, \\
& \vec{R}_{b \mathbf{m} a \mathbf{n}}(\vec{k})=e\left\langle\mathbf{m}\left|\left[\left\langle\vec{k}|\vec{r}| \phi_{b a}^{\mathrm{D}}\right\rangle+\langle\vec{k}| \vec{\phi}_{b a}^{\mathrm{C}}\right)\right] \mid \mathbf{n}\right\rangle \\
&\left|\vec{\phi}_{b a}^{\mathrm{C}}\right\rangle=\sqrt{N}\left\langle X_{b}\left|\sum_{i}^{N-1} \vec{r}_{i}\right| \Phi_{a}\right\rangle \\
& C_{a \mathbf{n}}(t)=\sum_{a^{\prime} \mathbf{n}^{\prime}} C_{a \mathbf{n} a^{\prime} \mathbf{n}^{\prime}}\left(t, t_{0}\right) C_{a^{\prime} \mathbf{n}^{\prime}}\left(t_{0}\right) \\
& C_{a^{\prime} \mathbf{n}^{\prime}}\left(t_{0}\right)=\left\langle\mathbf{n}^{\prime}\right|\left\langle\Phi_{a^{\prime}} \mid \Psi\left(\vec{r}, \vec{q}, t_{0}\right)\right\rangle \\
& C_{a \mathbf{n} a^{\prime} \mathbf{n}^{\prime}}\left(t, t_{0}\right) \\
&=e^{i E_{\mathrm{N}}\left(t-t_{0}\right) / \hbar} e^{i \epsilon_{\mathbf{n}^{\prime}} t_{0} / \hbar} e^{-i \epsilon_{\mathbf{n}} t / \hbar}\left\langle\mathbf{n}\left|\left\langle\Phi_{a}\left|\hat{U}_{0}\left(t, t_{0}\right)\right| \Phi_{a^{\prime}}\right\rangle\right| \mathbf{n}^{\prime}\right\rangle,
\end{aligned}
$$

$$
\begin{aligned}
& D_{b^{\prime} \mathbf{m}^{\prime} b \mathbf{m}}\left(t, t_{1}\right) \\
& \quad=e^{i E_{\mathrm{I}}\left(t-t_{1}\right) / \hbar} e^{i \epsilon_{\mathbf{m}} t_{1} / \hbar} e^{-i \epsilon_{\mathbf{m}^{\prime}} t / \hbar}\left\langle\mathbf{m}^{\prime}\left|\left\langle X_{b^{\prime}}\left|\hat{U}_{\mathrm{I}}\left(t, t_{1}\right)\right| X_{b}\right\rangle\right| \mathbf{m}\right\rangle,
\end{aligned}
$$

$$
\begin{gathered}
\phi_{d}\left(\vec{k}, t, t_{1}\right) \\
=\frac{I_{\mathrm{p}}}{\hbar}\left(t-t_{1}\right)+\frac{1}{2 m \hbar} \int_{t_{1}}^{t} d t^{\prime}\left(\hbar \vec{k}-e \vec{A}\left(t_{1}\right)+e \vec{A}\left(t^{\prime}\right)\right)^{2}, \\
I_{\mathrm{p}}=E_{\mathrm{I}}-E_{\mathrm{N}} .
\end{gathered}
$$

In Eq. (18), $\vec{R}_{b \mathbf{m} a \mathbf{n}}(\vec{k})$ is the dipole matrix element for a bound to continuum vibronic transition. The first term in Eq. (18) $\left(\left\langle\vec{k}|\vec{r}| \phi_{b a}^{\mathrm{D}}\right\rangle\right)$ arises due to the direct interaction between laser field and the active electron. The second contribution $\left(\left\langle\vec{k} \mid \vec{\phi}_{b a}^{\mathrm{C}}\right\rangle\right)$ is due to exchange-mediated interaction of the laser field with the inactive core electrons [108-110]. It is described by the three-component "cradle" vector orbital of Eq. (19) (after Newton's cradle, where a force acting on one ball in a multiball pendulum causes a different ball to swing [106]). Coefficients $C_{a \mathbf{n}}(t)$ describe the vibronic wave packet on the neutral surface at time $t$. Coefficients $C_{a \mathbf{n} a^{\prime} \mathbf{n}^{\prime}}\left(t, t_{0}\right)$ and $D_{b^{\prime} \mathbf{m}^{\prime} b \mathbf{m}}\left(t, t_{1}\right)$ are respectively the matrix elements of the neutral and ionic propagators $\hat{U}_{0}\left(t, t_{0}\right)$ and $\hat{U}_{\mathrm{I}}\left(t, t_{1}\right)$, where $\epsilon_{\mathbf{n}}$ is the energy of harmonic vibrational state $|\mathbf{n}\rangle$. The explicit form of the arbitrary-order Taylor expansion of these matrix elements was given in Ref. [90] and need not be repeated here. Equivalently, these matrix elements can also be obtained 
with MCTDH time propagation [91-95,97]. The phase factors containing $\epsilon_{\mathbf{n}}$ in Eqs. (22) and (23) arise to compensate for the presence of the vibrational phase factor in the definition of the wave-function ansatz in Ref. [90] [cf. Eq. (2) of Ref. [90]]. In Eqs. (22) and (23), we have extracted the rapidly oscillating overall phase of the matrix elements, using $E_{\mathrm{N}}$ and $E_{\mathrm{I}}$ as the characteristic energy of the neutral and cationic manifolds, respectively. Finally, $\phi_{d}$ [Eq. (24)] is the rapidly varying part of the HHG dipole phase accumulated from $t_{1}$ to $t$. The first term is due to the bound-state dynamics in the cation, with $I_{\mathrm{p}}$ being the characteristic ionization potential; the second contribution is the Volkov phase of the continuum electron.

Applying the usual stationary-phase approximation $[102,103]$ for the $\vec{k}$ integral in Eq. (17), we obtain

$$
\begin{aligned}
& \vec{d}(t)= \int_{t_{0}} d t_{1} \sum_{a^{\prime \prime} \mathbf{n}^{\prime \prime}} \sum_{b^{\prime} \mathbf{m}^{\prime}} \sum_{b \mathbf{m}} \sum_{a^{\prime \prime \prime} \mathbf{n}^{\prime \prime \prime}} \frac{1}{\hbar}\left(\frac{i 2 \pi m}{\hbar\left(t-t_{1}\right)}\right)^{3 / 2} \\
& \times C_{a^{\prime \prime} \mathbf{n}^{\prime \prime}}^{*}(t) \vec{R}_{b^{\prime} \mathbf{m}^{\prime} a^{\prime \prime} \mathbf{n}^{\prime \prime}}^{*}\left(\vec{k}_{\mathrm{s}}-\frac{e}{\hbar} \vec{A}\left(t_{1}\right)+\frac{e}{\hbar} \vec{A}(t)\right) \\
& \times D_{b^{\prime} \mathbf{m}^{\prime} b \mathbf{m}}\left(t, t_{1}\right) \vec{F}\left(t_{1}\right) \cdot \vec{R}_{b \mathbf{m} a^{\prime \prime \prime} \mathbf{n}^{\prime \prime}}\left(\vec{k}_{\mathrm{s}}\right) C_{a^{\prime \prime \prime} \mathbf{n}^{\prime \prime \prime}}\left(t_{1}\right) \\
& \times e^{-i \phi_{d}\left(\vec{k}_{\mathrm{s}}, t, t_{1}\right)}+\text { c.c. }, \\
& \vec{k}_{\mathrm{s}}\left(t, t_{1}\right)=-\frac{e}{\hbar\left(t-t_{1}\right)} \int_{t_{1}}^{t} \vec{A}\left(t^{\prime}\right) d t^{\prime}+\frac{e}{\hbar} \vec{A}\left(t_{1}\right),
\end{aligned}
$$

where $\vec{k}_{\mathrm{S}}$ is the stationary electron momentum at the time of ionization $t_{1}$.

Direct application of the stationary-phase approximation to the $d t_{1}$ integral in Eq. (26) leads to a generally complex stationary ionization time $t_{\mathrm{s}}$, causing difficulties when the matrix elements of Eq. (18) are only known numerically, on the the real axis. An alternative, real treatment is based on an observation that for all but the smallest $\Delta t=t-t_{1}$ values, the phase $\phi_{d}$ [Eq. (24)] is dominated by the Volkov phase $[98,102,103]$. If the term containing the $I_{\mathrm{p}}$ can be neglected and the field is linearly polarized, the stationary ionization time $t_{\mathrm{s}}$ is then determined by the condition $\vec{k}_{\mathrm{s}}\left(t, t_{\mathrm{s}}\right)=0$. Small, nonzero $I_{\mathrm{p}}$ values can then be accommodated using a Taylor expansion around the $\vec{k}_{\mathrm{s}}=0$ point [98]. For elliptically polarized fields, $\vec{k}_{\mathrm{s}}=0$ can not satisfy Eq. (27), as a small transverse initial momentum is required to bring the stationary trajectory back to the origin. The general condition determining the stationary ionization time then becomes

$$
\vec{k}_{\mathrm{s}} \cdot \frac{\partial \vec{A}(t)}{\partial t_{\mathrm{s}}} \equiv-\vec{k}_{\mathrm{s}} \cdot \vec{F}\left(t_{\mathrm{s}}\right)=0 .
$$

Equation (28) together with Eq. (27) define the real ionization time $t_{\mathrm{s}}$.

An additional complication arises when the vibronic wave function is even with respect to the origin and the laser field is linearly polarized. Then, the matrix element $\left.\vec{R}_{b \mathbf{m} a^{\prime \prime \prime} \mathbf{n}^{\prime \prime \prime}} \vec{k}_{\mathrm{s}}\right)$ in Eq. (26) vanishes at $\vec{k}_{\mathrm{s}}=0$, and the next order in the power series determines the overall integral over $d t_{1}$. Expanding the ionization dipole through the first order around $\vec{k}_{\mathrm{s}}$ and assuming that the remaining matrix elements in Eq. (26) vary slowly with $\vec{k}_{\mathrm{s}}$, we obtain (also see Appendixes A and B)

$$
\begin{aligned}
& \vec{d}(t)=\sum_{t_{\mathrm{s}}} \sum_{a^{\prime \prime} \mathbf{n}^{\prime \prime}} \sum_{b^{\prime} \mathbf{m}^{\prime}} \sum_{b \mathbf{m}} \sum_{a^{\prime \prime \prime} \mathbf{n}^{\prime \prime \prime}}\left(\frac{i 2 \pi m}{\hbar\left(t-t_{\mathrm{S}}\right)}\right)^{3 / 2} C_{a^{\prime \prime} \mathbf{n}^{\prime \prime}}^{*}(t) \\
& \times \vec{R}_{b^{\prime} \mathbf{m}^{\prime} a^{\prime \prime} \mathbf{n}^{\prime \prime}}^{*}\left(\vec{k}_{\mathrm{s}}-\frac{e}{\hbar} \vec{A}\left(t_{\mathrm{s}}\right)+\frac{e}{\hbar} \vec{A}(t)\right) D_{b^{\prime} \mathbf{m}^{\prime} b \mathbf{m}}\left(t, t_{\mathrm{s}}\right) \\
& \times \Upsilon_{b \mathbf{m} a^{\prime \prime \prime} \mathbf{n}^{\prime \prime \prime}}\left(\vec{k}_{\mathrm{s}}, t_{\mathrm{s}}\right) C_{a^{\prime \prime \prime} \mathbf{n}^{\prime \prime \prime}}\left(t_{\mathrm{s}}\right) e^{-i \phi_{d}\left(\vec{k}_{\mathrm{s}}, t, t_{\mathrm{s}}\right)}+\text { c.c. } \\
& \Upsilon_{b \mathbf{m} a^{\prime \prime \prime} \mathbf{n}^{\prime \prime \prime}}=\vec{F}\left(t_{\mathrm{s}}\right) \cdot \vec{R}_{b \mathbf{m} a^{\prime \prime \prime} \mathbf{n}^{\prime \prime \prime}}\left(\vec{k}_{\mathrm{s}}\right) 2 \pi\left(\frac{2 m}{e^{2} \hbar^{2} \vec{F}^{2}\left(t_{\mathrm{s}}\right)}\right)^{1 / 3} \operatorname{Ai}(\zeta) \\
& -2 i \pi \frac{\partial}{\partial \vec{k}_{\mathrm{s}}}\left[\vec{F}\left(t_{\mathrm{s}}\right) \cdot \vec{R}_{b \mathbf{m} a^{\prime \prime \prime} \mathbf{n}^{\prime \prime \prime}}\left(\vec{k}_{\mathrm{s}}\right)\right] \cdot\left(\frac{\hbar \vec{k}_{\mathrm{s}}}{t-t_{\mathrm{s}}}-e \vec{F}\left(t_{\mathrm{s}}\right)\right) \\
& \times\left(\frac{2 m}{e^{2} \hbar^{2} \vec{F}^{2}\left(t_{\mathrm{s}}\right)}\right)^{2 / 3} \mathrm{Ai}^{\prime}(\zeta) \\
& \zeta=\left(\frac{2 m}{e^{2} \hbar^{2} \vec{F}^{2}\left(t_{\mathrm{s}}\right)}\right)^{1 / 3}\left(E_{b \mathbf{m}}-E_{a^{\prime \prime \prime} \mathbf{n}^{\prime \prime \prime}}+\frac{\hbar^{2} \vec{k}_{\mathrm{s}}^{2}}{2 m}\right), \\
& E_{b \mathbf{m}}=\left\langle\mathbf{m}\left|\left\langle X_{b}\left|\hat{H}_{\mathrm{I}}\right| X_{b}\right\rangle\right| \mathbf{m}\right\rangle, \\
& E_{a^{\prime \prime \prime} \mathbf{n}^{\prime \prime}}=\left\langle\mathbf{n}^{\prime \prime \prime}\left|\left\langle\Phi_{a^{\prime \prime \prime}}\left|\hat{H}_{0}\right| \Phi_{a^{\prime \prime \prime}}\right\rangle\right| \mathbf{n}^{\prime \prime \prime}\right\rangle,
\end{aligned}
$$

where $E_{a^{\prime \prime \prime} \mathbf{n}^{\prime \prime}}$ and $E_{b \mathbf{m}}$ are respectively energies of the neutral and cationic vibronic basis functions. In Eq. (30) we neglected the explicit time dependence of $\vec{F}$, which is in the same order as other terms omitted in deriving Eq. 30 (see Appendix A). We have also neglected the $\vec{k}_{\mathrm{s}}$ dependence of the recombination

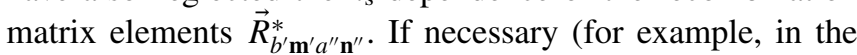
vicinity of a Cooper minimum), this dependence can be reintroduced in a similar manner.

Equation (29) is our final working equation. A closely related special-case result was previously obtained by Chirilă and Lein [65], and by Madsen et al. [86], which however did not consider the possibility of state crossings and nonadiabatic vibronic coupling. The special-case treatment of $\mathrm{SF}_{6}$ by Walters et al. [83] is similar in spirit, and in principle includes all effects considered here. Another closely related result by Faisal [84] includes rotational motion (neglected here), but restricts vibrational dynamics to a single surface. In contrast, the treatment of Ref. [88], while superficially similar, completely neglects all subcycle effects arising due to the motion on the intermediate cationic surfaces. These effects are also completely neglected by Ref. [87], which however treats the vibronic dynamics in the neutral manifold. Finally, treatments of Patchkovskii and Schuurman [90], Varandas et al. [91-95], and Arnold et al. [97] fully treat cationic vibronic dynamics, but neglect neutral dynamics and their coupling to the HHG process.

\section{COMPUTATIONAL DETAILS}

\section{A. Electronic structure calculations}

All electronic structure calculations used a correlationconsistent, valence triple- $\zeta$ basis set, augmented with diffuse functions ("aug-cc-pVTZ" [111,112]). Calculations of the neutral $\mathrm{CH}_{4}$ geometry and vibrational Hessian used secondorder Møller-Plesset (MP2) correlation treatment, with the $1 s$ 


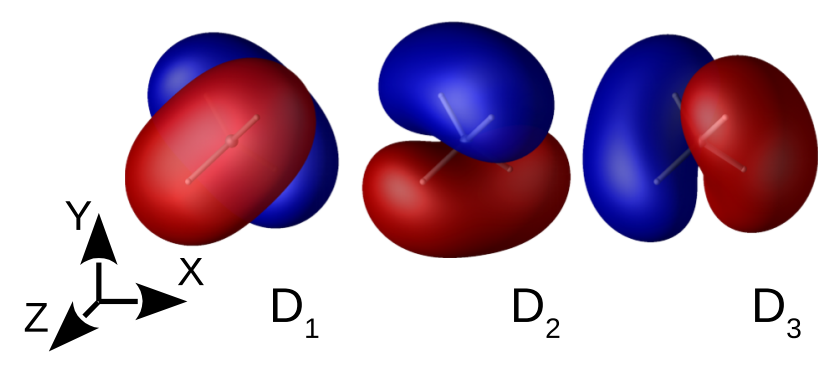

FIG. 1. MR-CIS Dyson orbitals for the three degenerate components of the lowest $\left({ }^{2} F_{2}\right)$ ionization channel in $\mathrm{CH}_{4}$. The orbitals are shown at the high-symmetry $T_{d}$ point. Isosurface levels are $0.02 a_{0}^{-3 / 2}$.

electrons on the carbon atom left uncorrelated. Optimized C-H bond length $\left(r_{0}=1.110 \AA\right)$ and unscaled harmonic vibrational frequencies $\left(v_{1} \ldots v_{4}=3069,1589,3204,1356 \mathrm{~cm}^{-1}\right)$ are in a good agreement with the experimental equilibrium geometry and fundamental frequencies $\left(r_{e}=1.094 \AA, v_{1} \ldots v_{4}=2916\right.$, $1534,3019,1306 \mathrm{~cm}^{-1}$ [113]).

The equilibrium neutral geometry corresponds to a symmetry-required triple degeneracy point of the ${ }^{2} F_{2}$ electronic ground state of the methane cation [114]. The electronic structure in the vicinity of this degeneracy point was explored by displacing all pairs of Cartesian coordinates by $\pm 0.02 \AA$, for the total of 450 unique distorted structures. The reference geometry was taken in the standard setting of the $T_{d}$ group, with the $C_{2}$ axes along the main Cartesian directions (see Fig. 1). Symmetry was used neither to reduce the number of the displaced geometries nor in the electronic structure calculations at these geometries.

Electronic wave functions of the three lowest cation states correlating to the ${ }^{2} F_{2}$ state were calculated using multireference configuration interaction with single excitations treatment (MR-CIS). All configurations within the minimal complete active space with seven electrons in four frontier orbitals $[\mathrm{CAS}(7,4)]$ were included in the reference set. Single-particle orbitals were optimized in a CAS $(7,4)$ self-consistent field (CASSCF) calculation, with the energies of the three cation states correlating to ${ }^{2} F_{2}$ weighted equally. The neutral wave functions were determined within the CIS space, using the lowest closed-shell determinant constructed from the cation-optimized orbitals as the reference. The MR-CIS vertical ionization potential (MR-CIS: $13.14 \mathrm{eV}$ ) slightly underestimates the experimental value (experiment: $13.60 \mathrm{eV}[115,116])$. Including double excitations in the CI expansion (MR-CISD; Langhoff-Davidson $+Q$ correction [117] not included) leads to an overestimation of the vertical IP by a similar amount (MR-CISD: $13.97 \mathrm{eV}$ ). Given the large number of the distorted geometries, further increase in the CI size is not practical. It is in principle possible to adjust the calculated vertical IP to match the experimental value. However, the experimental vertical ionization potential is somewhat uncertain, with a very, very broad, complex peak in the 13.2-14.0-eV range [116,118]. Because all qualitative features of the energy surfaces are already adequately described at the MR-CIS level, all subsequent calculations use MR-CIS wave functions and energies. Due to the underestimation of the vertical IP, we expect ionization rates [Eq. (30)] to be somewhat overestimated.

\section{B. Diabatization procedure}

Evaluation of matrix elements entering Eq. (29) requires that both the potential energy surfaces and the wave functions are smooth and continuous in the vicinity of the expansion point. It is therefore necessary to diabatize the MR-CIS states. Construction of quasidiabatic states is a long-standing and still active area of research (see Ref. [63] for an overview), with many competing prescriptions available in the literature. An appealing, generally applicable approach for constructing a local quasidiabatic surfaces involves fitting adiabatic energy gradients and derivative couplings at selected points in the nuclear coordinate space [119-121]. Unfortunately, this technique does not offer an easy access to the transformed quasidiabatic wave functions, which are needed for evaluating the somewhat nonstandard matrix elements $\Upsilon$ and $\vec{R}$ [Eqs. (18) and (30)]. Here, we retain the quadratic vibronic Hamiltonian form of Ref. [119], but revert to the older maximum-overlap approach [122-124] to directly determine the diabatic wave functions:

$$
\Psi_{j a}^{\mathrm{dia}}=\sum_{i} U_{j i a} \Psi_{i a}^{\mathrm{ad}},
$$

where $\Psi_{j a}^{\mathrm{dia}}$ is the $j$ th quasidiabatic state at geometry $a, \Psi_{i a}^{\mathrm{ad}}$ is the $i$ th adiabatic state at the same geometry. The unitary transformation $U_{j i a}$ connects the two wave-function spaces. At each displaced geometry $(a)$, the optimal matrix $\mathbf{U}_{a}$ is determined [122-127] by the overlap matrix $\mathbf{S}_{a}$ between the adiabatic wave functions at the displaced geometry and reference wave functions $\Psi_{k}^{\text {ref }}$ (see Fig. 1):

$$
\begin{gathered}
S_{i k a}=\left\langle\Psi_{i a}^{\mathrm{ad}} \mid \Psi_{k}^{\mathrm{ref}}\right\rangle, \\
\mathbf{U}_{a}^{*}=\left(\mathbf{S}_{a}^{\dagger} \mathbf{S}_{a}\right)^{-1 / 2} \mathbf{S}_{a}^{\dagger} .
\end{gathered}
$$

For methane, reference wave functions $\Psi^{\text {ref }}$ are taken at the high-symmetry $T_{d}$ geometry, treated within the $D_{2}$ subgroup. The neutral manifold uses the ${ }^{1} A_{1}$ ground-state wave function, while the cationic manifold is referenced to the lowest ${ }^{2} B_{1}$, ${ }^{2} B_{2}$, and ${ }^{2} B_{3}$ wave functions.

Once the diabatization transformation $\mathbf{U}_{a}$ is determined, the quadratic vibronic Hamiltonian is obtained with a leastsquares fit to the diabatic Hamiltonian matrices at the displaced geometries. Electronic matrix elements are calculated directly from the transformed diabatic wave functions [Eq. (34)] as described elsewhere [110,128] (also see Appendix C). For the methane molecule in the vicinity of the equilibrium neutral geometry, the resulting vibronic Hamiltonian coincides with the result of [119], while the residual derivative couplings are found to be numerically negligible. Direct nonlinear minimization of the quadratic vibronic Hamiltonian fit with respect to the diabatization parameters $\mathbf{U}_{a}$ leaves the result unchanged, confirming that the transformation of Eq. (36) represents a local optimum.

It should be noted that the quadratic vibronic Hamiltonian of the ${ }^{2} F_{2}$ manifold of $\mathrm{CH}_{4}{ }^{+}$determined presently focuses on the small region of configuration space in the vicinity of the neutral equilibrium geometry. It is unbound from below, and corresponds to a dissociative state. As a result, it is not capable of describing long-time dynamics of the cation, and should not 


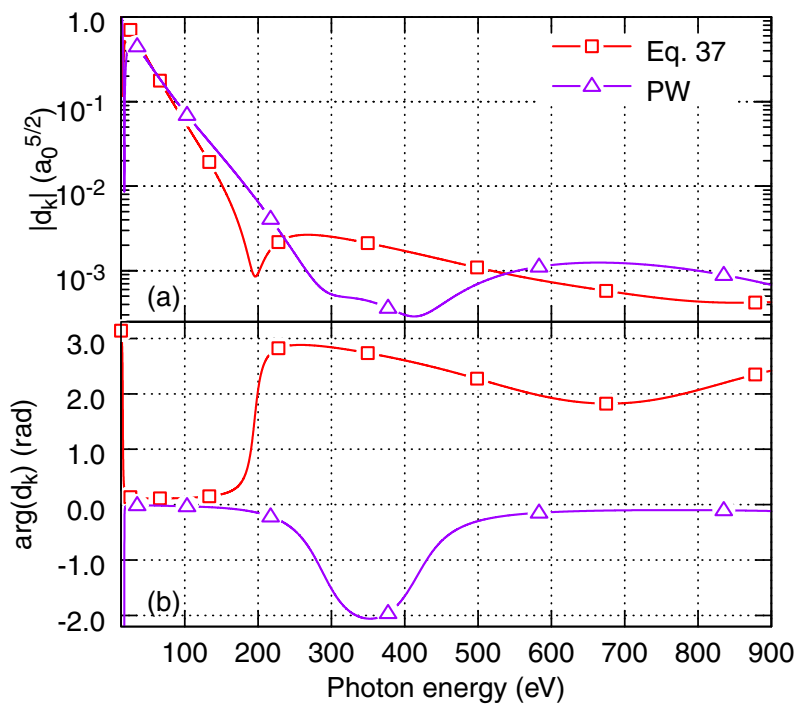

FIG. 2. Magnitude (a) and phase (b) of the dipole photoionization matrix elements [Eq. (37)] for the $D_{3}$ component of the ${ }^{2} F_{2}$ ionization channel at the high-symmetry point (Fig. 1). The $\vec{k}$ vector is taken along the positive $X$ Cartesian direction. The cradle contributions [Eq. (19)] are significant beyond $\approx 150 \mathrm{eV}$. Omitting the cradle terms leads to the "plane-wave" (PW) curve.

be compared globally to the more conventional, spectroscopic surfaces, such as used in Refs. [91,92].

\section{Dyson orbitals and dipole matrix elements}

Dyson orbitals [Eq. (13)] corresponding to the ground-state $\left({ }^{2} F_{2}\right)$ ionization channel of the $\mathrm{CH}_{4}$ molecule are shown in Fig. 1. All three components are strongly one-electron allowed, with a Dyson orbital norm of 0.916. Visually, these Dyson orbitals are indistinguishable from the triply degenerate highest occupied molecular orbital of the neutral species. The dominant "cradle" terms [Eq. (19), not shown] are derived from the ( $s$-like) $2 a_{1}$ molecular orbital of the methane molecule.

It is instructive to examine the electronic part of the photoionization matrix elements:

$$
\vec{d}_{e}=e\left[\left\langle\vec{k}|\vec{r}| \phi_{b a}^{\mathrm{D}}\right\rangle+\left\langle\vec{k} \mid \vec{\phi}_{b a}^{\mathrm{C}}\right\rangle\right]
$$

[see Eq. (18)] a little more closely. Due to the high symmetry of the $\mathrm{CH}_{4}$ molecule, it is sufficient to constrain the photoelectron observation direction to one of the Cartesian axes (see Figs. 1 and 2). The dominant first term in the matrix element is identical to the first Born approximation in photoionization, known to be qualitatively inaccurate close to the ionization threshold. Beyond $30 \mathrm{eV}$ photon energy, however, the calculated cross sections are in (fortuitously) good agreement with experimental data [116,129-133] and accurate calculations [134-136]. For example, Eq. (37) yields a photoionization cross section of $\approx 17$ Mbarn at $30 \mathrm{eV}$ photon energy (experiment: $\approx 14$ Mbarn [136]), decreasing to $\approx 5$ Mbarn at $60 \mathrm{eV}$ (experiment: $\approx 2$ Mbarn [136]), and to $\approx 1.2$ Mbarn at $90 \mathrm{eV}$ (experiment: $\approx 0.6$ Mbarn [136]).

The cradle terms become important [108] beyond $\approx 150 \mathrm{eV}$ photon energy (see Fig. 2), leading to an $\approx 0.4$-kbarn minimum in the calculated cross sections at $\approx 196 \mathrm{eV}$. It is followed by a maximum $(\approx 7 \mathrm{kbarn})$ at $295 \mathrm{eV}$ (again fortuitously) close to where the cross section is expected to increase due to the $K$-edge intensity borrowing [104]. Unfortunately, we are not aware of reliable final-state resolved calculations or measurements in methane for photon energies above $100 \mathrm{eV}$, where ionization from the inner $2 a_{1}$ and $1 a_{1}$ shells dominates the overall cross section [116]. It is therefore unclear whether the photoionization matrix elements in Fig. 2 provide a reasonable description of methane photoionization beyond $90 \mathrm{eV}$. Nonetheless, the minimum and the associated $\pi$ phase jump at $196 \mathrm{eV}$ serve to illustrate an important point (see below), which remains qualitatively valid even it occurs at a different energy in the actual $\mathrm{CH}_{4}$ molecule than in our crude calculations here.

\section{Nuclear autocorrelation and MC-SFA-GWP HHG calculations}

Nuclear autocorrelation functions are a special case of the vibronic matrix elements $D_{b^{\prime} \mathbf{m}^{\prime} b \mathbf{m}}(t, 0)$ of Eq. (23), with $b^{\prime}=b$ and $m^{\prime}=m$. Both $D_{b^{\prime} \mathbf{m}^{\prime} b \mathbf{m}}$ and $C_{a^{\prime \prime \prime} \mathbf{n}^{\prime \prime \prime}}$ matrix elements are propagated in time numerically, using the fourth-order RungeKutta integrator with a uniform time step [137]. Calculations of the autocorrelation function used a time step of $0.02 \mathrm{au}[\mathrm{t}]$ $(\approx 0.48$ as). Short-time autocorrelation functions calculated presently numerically coincide with the analytical results [90], for all times where the power-series expansion in Ref. [90] converges.

Calculations of the time-dependent MC-SFA-GWP dipole $\vec{d}(t)$ [Eq. (29)] used a time step corresponding to $1 / 3$ of the Nyquist limit for the cutoff harmonics. The resulting time step ranged from $0.386 \mathrm{au}[\mathrm{t}](\approx 9.3 \mathrm{as})$ for the 800 -nm, $300-\mathrm{TW} \mathrm{cm}^{-2}$ driving field, to $0.037 \mathrm{au}[\mathrm{t}](\approx 0.89$ as) for the $1.6-\mu \mathrm{m}, 1-\mathrm{PW} \mathrm{cm}{ }^{-2}$ driver. We did verify that all calculations are converged with respect to the time step. The high-orderharmonic spectrum was calculated from the Fourier transform of the time-dependent dipole $[102,103]$. No window function was applied.

The vibronic wave-function expansion was performed in the basis of multidimensional harmonic oscillator functions of the neutral species. Rotational and translational modes were excluded. Calculations of the autocorrelation functions allowed up to 12 quanta in any of the remaining nine normal modes. This choice is fully converged with respect to the basis set up to at least 4.5 fs evolution time, but is too computationally expensive for MC-SFA-GWP calculations, where the vibronic matrix elements need to be recalculated for each trajectory. Instead, MC-SFA-GWP calculations limited the vibronic basis to at most four quanta in any of the vibrational modes. This choice changes the autocorrelation function by less than $0.2 \%$ at 1.7 fs delay $(800-\mathrm{nm}$ cutoff trajectory), increasing to $3 \%$ at $2.6 \mathrm{fs}$ (1200-nm cutoff) and $25 \%$ at 3.4 fs (1600-nm cutoff). Our MC-SFA-GWP results are therefore sufficiently converged at 800 and $1200 \mathrm{~nm}$, but are only qualitative for the high-energy part of the spectrum at $1800 \mathrm{~nm}$ (beyond 160/500 eV at 300/1000 $\mathrm{TW} \mathrm{cm}^{-2}$ ).

All MC-SFA-GWP calculations were performed for laser electric field polarized along the molecular $X$ axis. Due to the high symmetry of the $\mathrm{CH}_{4}$ molecule, we expect orientational 


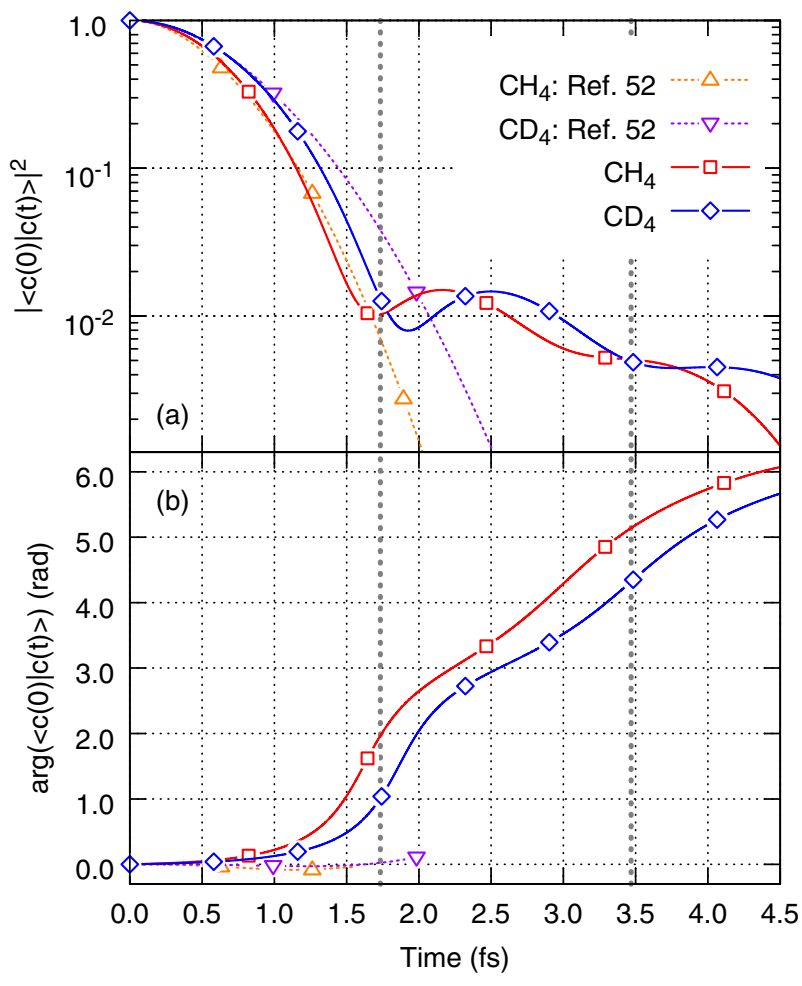

FIG. 3. Nuclear autocorrelation function in methane cation. Initial nuclear wave packet $|c(0)\rangle$ is the ground vibrational state of the neutral species. Solid red line: $\mathrm{CH}_{4}$, this work. Solid blue line: $\mathrm{CD}_{4}$, this work. Dotted orange line: $\mathrm{CH}_{4}$ using field-diabatized cationic energy surface (Ref. [52]). Dotted purple line: $\mathrm{CD}_{4}$ from Ref. [52]. Panel (a) shows the squared absolute magnitude of the autocorrelation function. Panel (b) gives the phase of the autocorrelation function. Linear phase due to the zero-point energy has been subtracted. Dotted, gray vertical lines indicate time delays for the cutoff trajectories for 800- and 1600-nm driving fields.

averaging to play only a minor role for this system. Only the short trajectories were considered.

\section{RESULTS AND DISCUSSION}

\section{A. Free-cation dynamics and autocorrelation function}

The simplest summary of the subcycle nuclear dynamics is provided by the nuclear autocorrelation function, shown in Fig. 3 for both $\mathrm{CH}_{4}$ and $\mathrm{CD}_{4}$ cations, using neutral vibrational ground state as the initial wave function at time zero. For comparison, we show autocorrelation functions for the same species and initial conditions from Ref. [52]. At very short times (up to $\approx 1.2 \mathrm{fs}$ ), all four autocorrelation functions are Gaussian in time [please note the linear-log scale of Fig. 3(a)], which is a generic feature of short-time autocorrelation functions [52,138]. The agreement between the data sets is remarkably good, considering the differences in the theoretical treatment. In Ref. [52], the autocorrelation functions are calculated on a single component of the ${ }^{2} F_{2}$ electronic surface, with the degeneracy lifted by applying an intense $\left(\approx 2.6 \mathrm{~V} \AA^{-1}\right)$ static electric field. Because the components of the ${ }^{2} F_{2}$ state are coupled by the electric field (MR-CIS transition dipole of $\approx 0.7 \mathrm{D}$ ), the surface shape is also modified, allowing the single-surface dynamics to mimic the more complex vibronic dynamics at early times.

At the same time, the single-surface treatment does not allow for the "orbiting" motion of the wave packet around the conical intersection point, precluding the possibility of revivals on a few-femtosecond time scale. Beyond $\approx 1.2 \mathrm{fs}$, a single, field-diabatized surface no longer adequately represents vibronic dynamics in this system. For $\mathrm{CH}_{4}\left(\mathrm{CD}_{4}\right)$, the population of the initial surface reaches a minimum of $52 \%$ $(51 \%)$ at $1.5 \mathrm{fs}(1.7 \mathrm{fs})$, then begins to increase again as a fraction of the wave packet completes a half revolution around the CI. Correspondingly, the autocorrelation function changes sign [see Fig. 3(b)] and undergoes a half revival at $2.15 \mathrm{fs}$ (2.50 fs). For both $\mathrm{CH}_{4}$ and $\mathrm{CD}_{4}$, the nuclear autocorrelation factor in HHG reaches $1.5 \%$ at the half revival-more than an order of magnitude above the expected damping factor due to the Gaussian decay [52]. A full wave packet revival appears as a shoulder at the $0.5 \%$ level, at $\approx 3.6 \mathrm{fs}(\approx 4.2 \mathrm{fs})$. Similar, nonGaussian intermediate-time decay and unexpected revivals have been previously predicted in other nonadiabatic systems [89,90,97].

The short-time autocorrelation functions in Fig. 3 are qualitatively consistent with the previously reported calculations by Mondal and Varandas [91,92]. These authors find the first half revival at $\approx 2.4$ fs for $\mathrm{CH}_{4}{ }^{+}\left(\approx 2.8\right.$ fs for $\left.\mathrm{CD}_{4}{ }^{+}\right)$. The full revivals are also found at times comparable to our results. At the same time, the maximum of the $\mathrm{CD}_{4} / \mathrm{CH}_{4}$ autocorrelation functions ratio calculated in Ref. [92] is much higher $(\approx 6$, compared to $\approx 2.7$ here). It is also reached at a later time $(\approx 1.85 \mathrm{fs}$, compared to $\approx 1.5 \mathrm{fs}$ here $)$. Both discrepancies are well within the range of available experimental resolution, and would be interesting to explore.

Furthermore, in contrast to Refs. [91,92], we cannot attribute the revivals to the dynamics reaching the Jahn-Teller minima on the cationic energy surface. Calculated expectation values of normal coordinates on each electronic surface evolve monotonically at least until $3.5 \mathrm{fs}$. The Cartesian displacements in the center-of-mass coordinate system remain small (less than $0.02 \AA$ ), with the structure remaining at the $D_{2 d}$ symmetry, indicating that the minimum is not reached at the times relevant for the PACER experiments at mid-IR wavelengths.

It is therefore instructive to examine the origin of the oscillations in the short-time autocorrelation function in a little more detail. The square modulus of the autocorrelation function measures the population of the initially populated vibronic basis function $\left(D_{1}|g s\rangle\right.$ in Fig. 4). The strongest vibrational coupling of $|\mathrm{gs}\rangle$ on the same quasidiabatic electronic surface is to a vibrational basis function with a single quantum in the $v_{2}$ vibrational mode $\left(\left|\nu_{2}\right\rangle: e\right.$ symmetry, $1589 \mathrm{~cm}^{-1}, \mathrm{H}-\mathrm{C}-\mathrm{H}$ bending motion). The strongest coupling to the other two quasidiabatic surfaces $\left(D_{2}\right.$ and $\left.D_{3}\right)$ involve single excitation of the $v_{4}\left(\left|v_{4}\right\rangle: t_{2}, 1356 \mathrm{~cm}^{-1}, \mathrm{H}-\mathrm{C}-\mathrm{H}\right.$ bend) and $v_{3}\left(\left|v_{3}\right\rangle: t_{2}, 3204 \mathrm{~cm}^{-1}, \mathrm{C}-\mathrm{H}\right.$ stretch) normal modes. All of these couplings are of a similar magnitude. The number of significant couplings increases rapidly with the vibrational excitation level; once two or more vibrational quanta have been excited, the probability of the wave packet returning to the initial state $D_{1}|\mathrm{gs}\rangle$ becomes negligible. 


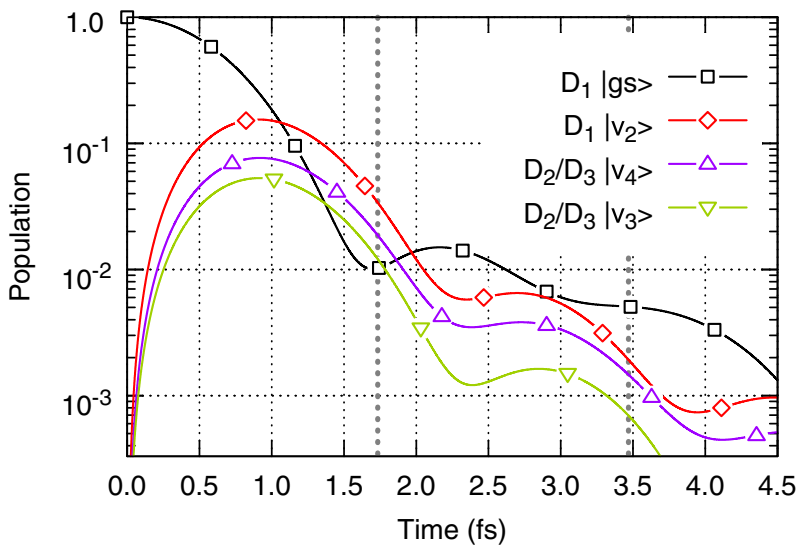

FIG. 4. Transient population of dominant singly excited vibronic basis functions in $\mathrm{CH}_{4}{ }^{+}$. The initially prepared wave packet is ground vibrational state of the neutral molecule, placed on the $D_{1}$ diabatic surface ( $D_{1}|\mathrm{gs}\rangle$, black squares). This vibronic basis function is directly coupled to the singly excited $v_{2}$ mode on the same electronic surface ( $D_{1}\left|v_{2}\right\rangle$, red diamonds), singly exited $v_{4}$ mode on the $D_{2}$ and $D_{3}$ surfaces $\left(D_{2} / D_{3}\left|v_{4}\right\rangle\right.$, purple triangles), and singly excited $\nu_{3}$ mode on the same surfaces $\left(D_{2} / D_{3}\left|\nu_{3}\right\rangle\right.$, green inverted triangles).

The short-time evolution of the population of the initially populated and singly excited vibronic basis functions in $\mathrm{CH}_{4}{ }^{+}$ is illustrated in Fig. 4. One can immediately see that the populations of all five dominant singly excited functions $\left(D_{1}\left|v_{2}\right\rangle, D_{2} / D_{3}\left|v_{4}\right\rangle\right.$, and $\left.D_{2} / D_{3}\left|v_{3}\right\rangle\right)$ oscillate in phase at least up to $4.5 \mathrm{fs}$. The oscillations are out of phase with the autocorrelation magnitude ( $D_{1}|g s\rangle$ population), indicating the back-and-forth population transfer. This behavior is easy to rationalize by considering a reduced-dimensionality model, consisting of just the six essential states listed above. The corresponding model Hamiltonian is

$$
H_{\text {mod, } 1}=\left(\begin{array}{llllll}
0 & b_{1} & b_{2} & b_{2} & b_{3} & b_{3} \\
b_{1} & d_{1} & & & & \\
b_{2} & & d_{2} & & 0 & \\
b_{2} & & & d_{2} & & \\
b_{3} & & 0 & & d_{3} & \\
b_{3} & & & & & d_{3}
\end{array}\right),
$$

where all elements not in the first row or column, or on the main diagonal, are zero. As long as all $d_{i} \approx d$, this Hamiltonian is equivalent to a two-level system:

$$
\begin{gathered}
H_{\text {mod }, 2}=\left(\begin{array}{ll}
0 & b_{0} \\
b_{0} & d
\end{array}\right), \\
b_{0}=\sqrt{b_{1}^{2}+2 b_{2}^{2}+2 b_{3}^{2}},
\end{gathered}
$$

where the transformed basis is

$$
\begin{aligned}
\phi_{1}= & D_{1}|\mathrm{gs}\rangle, \\
\phi_{2}= & b_{0}^{-1}\left\{b_{1} D_{1}\left|v_{2}\right\rangle+b_{2} D_{2}\left|v_{4}\right\rangle+b_{2} D_{3}\left|v_{4}\right\rangle\right. \\
& \left.+b_{3} D_{2}\left|v_{3}\right\rangle+b_{3} D_{3}\left|v_{3}\right\rangle\right\} .
\end{aligned}
$$

The $\phi_{2}$ basis function corresponds to a vibronic wave packet component "orbiting" the conical intersection. In the nuclear coordinate space, $\left|\phi_{2}\right\rangle$ forms a prolate spheroidal shell around the conical intersection. The oscillatory behavior in the autocorrelation function (i.e., the population of the $\left|\phi_{1}\right\rangle$ basis function) is due to the interference between the two eigenstates of Eq. (39), while the overall decay of the signal in this model arises from the imaginary part of $d[\operatorname{Im}(d)<0]$. To the order $O\left(d^{2}\right)$, the difference between the eigenvalues of $H_{\text {mod,2 }}$ is

$$
\Delta E_{\mathrm{mod}, 2} \approx 2 b_{0}
$$

Thus, the oscillation frequency of the short-time autocorrelation function is determined by the strength of the coupling between the initially prepared component of the wave packet and its decaying part orbiting the conical intersection.

As can be clearly seen from Eq. (40), nonadiabatic vibronic coupling between the electronic surfaces plays a key role in the initial wave-packet decay. If vibronic coupling is neglected, the initial decay is slowed down dramatically (data not shown), as was seen before for the benzene cation [90]. The resulting lower isotope effects are no longer compatible with experimental data (see Sec. IV C below). A similar observation was made in Ref. [86], which neglected the vibronic coupling.

Coordinate dependence of the tunneling-ionization matrix elements [Eq. (30)] can lead to substantial reshaping of the neutral vibrational wave packet upon ionization [139]. In methane, ionization primarily populates $v_{4}$ (asymmetric bend) and $v_{3}$ (asymmetric stretch) modes. Nuclear autocorrelation functions for single-quantum excitation of these two modes in $\mathrm{CH}_{4}$ and $\mathrm{CD}_{4}$ are shown in Fig. 5. These autocorrelations are qualitatively similar to the case of the vibrational ground state (Fig. 3). However, the first half revival occurs at an earlier time,

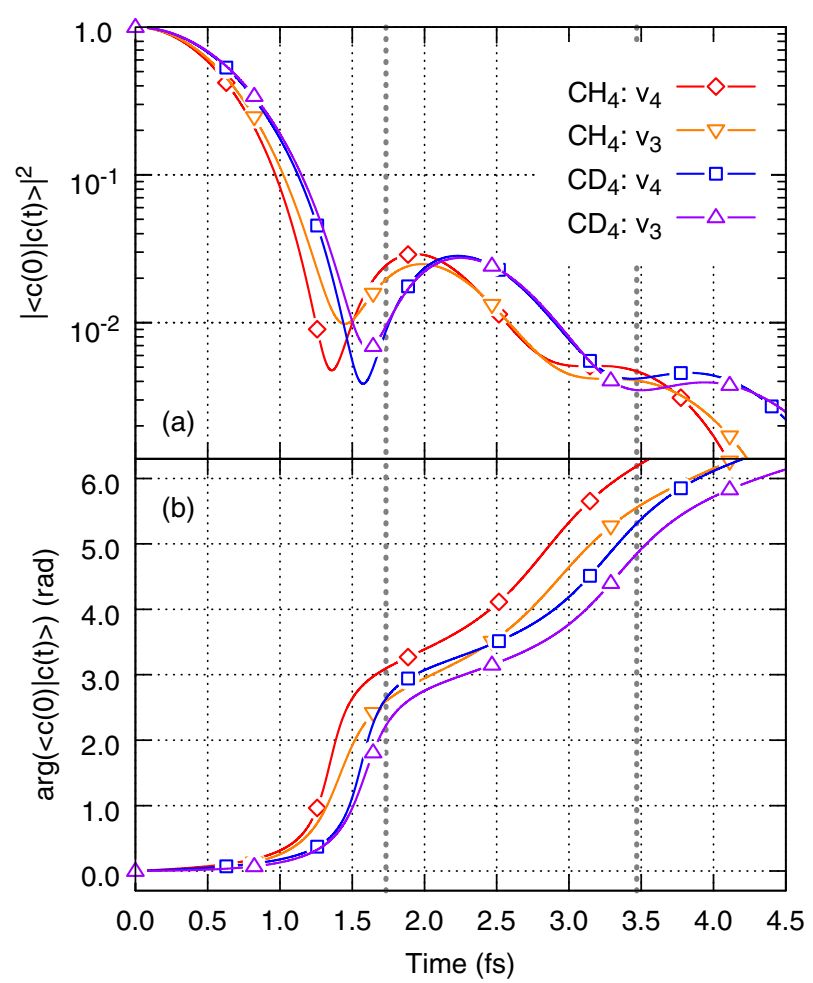

FIG. 5. Nuclear autocorrelation function in vibrationally excited methane cation. Initial nuclear wave function $|c(0)\rangle$ has a single vibrational quantum in the asymmetric bend $\left(v_{4}, 1356 / 1028 \mathrm{~cm}^{-1}\right.$ in $\left.\mathrm{CH}_{4} / \mathrm{CD}_{4}\right)$ or asymmetric stretch $\left(v_{3}, 3204 / 2379 \mathrm{~cm}^{-1}\right)$ vibrational modes. Panels and the scale of the plots is the same as in Fig. 3; see Fig. 3 caption for further details. 
and has a substantially higher magnitude $\left(\mathrm{CH}_{4}: 2.9 / 2.5 \%\right.$ at $1.93 / 1.98$ fs for $v_{4} / v_{3} ; \mathrm{CD}_{4}: 2.8 / 2.7 \%$ at $2.23 / 2.25$ fs for $v_{4} / v_{3}$ respectively). The faster short-time dynamics of vibrationally excited states has been noted before $[52,65,67,85,94]$. This property will be important for understanding the HHG spectra at longer wavelengths (Sec. IV B below).

Overall, nonadiabatic nuclear autocorrelation factors suggest that HHG emission in methane should persist at much longer times than expected previously. Even at $4.3 \mathrm{fs}$, close to the short-trajectory cutoff for a $2-\mu \mathrm{m}$ IR driver, the autocorrelation factor remains above $0.2 \%\left(0.4 \%\right.$ for $\left.\mathrm{CD}_{4}\right)$-within the possible measurement range. Furthermore, the nonadiabatic dynamics around the CI can be expected to lead to a rich PACER signal, including inverse isotope effects between 1.8 and $2.3 \mathrm{fs}$. A more detailed investigation of the HHG signal therefore appears justified, and is attended to in Secs. IV B and IV $\mathrm{C}$ below.

\section{B. High-order-harmonic generation}

For calculations of high-order-harmonic spectra, we consider three driver wavelengths $(800 \mathrm{~nm}, 1.2 \mu \mathrm{m}$, and $1.6 \mu \mathrm{m})$ and two peak intensities $\left(300 \mathrm{TW} \mathrm{cm} \mathrm{m}^{-2}\right.$ and $\left.1 \mathrm{PW} \mathrm{cm}^{-2}\right)$. For each combination of the wavelength, intensity, and isotopic species, we perform four simulations, as follows.

FS: In this simulation, the nuclei are "frozen," and are not allowed to move between ionization and recollision. To this end, the vibronic matrix elements of Eqs. (22) and (23) are replaced by

$$
\begin{aligned}
C_{a \mathbf{n} a^{\prime} \mathbf{n}^{\prime}}^{\text {froen }}\left(t, t_{0}\right) & =e^{i E_{\mathrm{N}}\left(t-t_{0}\right) / \hbar}, \\
D_{b^{\prime} \mathbf{m}^{\prime} b \mathbf{m}}^{\text {frozen }}\left(t, t_{1}\right) & =e^{i E_{\mathrm{I}}\left(t-t_{1}\right) / \hbar} .
\end{aligned}
$$

All other matrix elements and the initial nuclear wave packet, represented by the $C_{a^{\prime} \mathbf{n}^{\prime}}\left(t_{0}\right)$ coefficients in Eq. (20), remain unchanged. As the result, this simulation can still exhibit isotope effects due to the differences in the initial ground-state wave packet widths combined with coordinate dependence of the matrix elements.

AC: In this "autocorrelation" simulation, the electronic part of the dipole is calculated exactly as in the frozen-nuclei simulation FS. For each trajectory, the dipole is multiplied by the magnitude of the nuclear autocorrelation function (Sec. IV A). Thus, electronic continuum and vibronic dynamics are assumed to be factorized and mutually independent. This is the the approach typically taken for the analysis of PACER spectra.

ND: In this "no-dipole" simulation, we use the full Eq. (29). However, the effects of the laser field are neglected when evaluating vibronic matrix elements of Eqs. (22) and (23). Thus, vibronic dynamics in the neutral species and the cation are taken to be field free, and depend only on the initial composition of the wave packet and the elapsed time. The laser field interacts with the continuum part of the wave function at all times, but with the rest of the molecule only at the moment of ionization, through the $\Upsilon_{b \mathbf{m} a^{\prime \prime \prime} \mathbf{n}^{\prime \prime \prime}}$ matrix element of Eq. (30). Electronic and nuclear dynamics are otherwise fully correlated.

DS: Finally, the "dipole" simulation uses Eq. (29), with no further simplifications to any of the matrix elements. The laser field can cause (subcycle) vibrational excitation in the parent
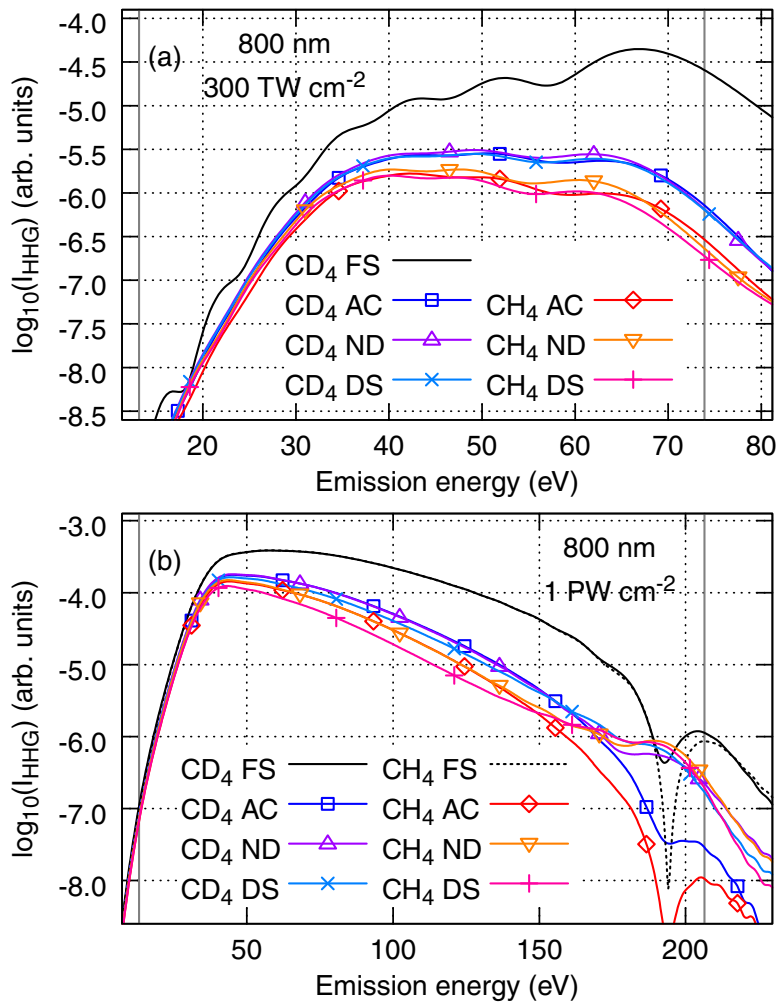

FIG. 6. MC-SFA-GWP high-order-harmonic spectrum of methane for a single-sided recollision in 800-nm driving field. Only short trajectories are included. The ionization potential $\left(I_{\mathrm{p}}\right)$ and the harmonic cutoff $\left(3.17 U_{\mathrm{p}}+1.3 I_{\mathrm{p}}\right)$ are indicated by vertical grey lines. The labels are as follows (see text for details): Nuclear geometry frozen, no nuclear factors included: $\mathrm{FS}\left(\mathrm{CD}_{4}\right.$ : solid black line; $\mathrm{CH}_{4}$ : dotted black line). Nuclear geometry frozen, autocorrelation factor included: $\mathrm{AC}\left(\mathrm{CD}_{4}\right.$ : blue; $\mathrm{CH}_{4}$ : red). MC-SFA-GWP, vibronic terms due to laser coupling omitted: $\mathrm{ND}\left(\mathrm{CD}_{4}\right.$ : purple; $\mathrm{CH}_{4}$ : orange). MC-SFA-GWP, all terms included: DS ( $\mathrm{CD}_{4}$ : teal; $\mathrm{CH}_{4}$ : magenta). Panel (a) $300 \mathrm{TW} \mathrm{cm}{ }^{-2}$. The $\mathrm{CD}_{4} \mathrm{FS}$ and $\mathrm{CH}_{4}$ FS (not shown) curves are visually indistinguishable. (b) $1 \mathrm{PW} \mathrm{cm}^{-2}$.

neutral species, modify the nuclear wave packet through the ionization matrix elements $\Upsilon_{b \mathbf{m} a^{\prime \prime \prime} \mathbf{n}^{\prime \prime \prime}}$, and cause vibronic transitions on the cationic surface. DS is our best-effort simulation.

Calculated normal and deuterated methane HHG spectra for the 800-nm driving field are shown in Fig. 6. At the moderate, $300 \mathrm{TW} \mathrm{cm}^{-2}$ intensity of the driving field [panel (a)], harmonic cutoff is found at $\approx 74 \mathrm{eV}$ photon energy (harmonic 47). For these field parameters, the frozen-nuclei spectra of the two isotopic species are indistinguishable. Close to the cutoff, nuclear motion leads to HHG suppression by a factor of $\approx 75 \times\left(\mathrm{CD}_{4}\right)$ or $100 \times\left(\mathrm{CH}_{4}\right)$. All three approaches we consider for treating the nuclear motion yield essentially the same results, indicating that the vibronic and recollision dynamics are largely uncorrelated, and the subcycle vibronic dynamics is not affected by the laser field.

The situation changes when the intensity is increased to $1 \mathrm{PW} \mathrm{cm}{ }^{-2}$ [Fig. 6(b)]. Now, the HHG cutoff extends to $\approx 206 \mathrm{eV}$ (H133), past the minimum in the recombination cross section (see Fig. 2). In the vicinity of the minimum, the finite width of the initial nuclear wave packet now leads to isotope dependence even in the absence of subcycle nuclear 
motion: $\mathrm{HHG}$ emission from $\mathrm{CD}_{4}\left(\mathrm{CD}_{4} \mathrm{FS}\right.$, solid black line) in the vicinity of the minimum is $\approx 55 \times$ stronger than for $\mathrm{CH}_{4}\left(\mathrm{CH}_{4} \mathrm{FS}\right.$, dotted black line). At a first glance, this result appears counterintuitive: the broader ground-state vibrational wave packet in $\mathrm{CH}_{4}$ is expected to sample a wider range of nuclear configuration, and thus better "fill in" the 196-eV structural minimum in the recombination matrix element. However, in the vicinity of the $\mathrm{CH}_{4}$ harmonic minimum $(\approx 194 \mathrm{eV})$, the phase of the recombination matrix element varies substantially over the characteristic extent of the ground-state nuclear wave function. The destructive interference then leads to a much stronger suppression in $\mathrm{CH}_{4}$ FS harmonic emission than might have been expected from the matrix elements at the equilibrium geometry alone. The narrower distribution of the nuclear positions in the heavier $\mathrm{CD}_{4}$ reduces the extent of the destructive interference, leading to increased HHG intensity near the minimum.

The qualitative features of the frozen-nuclei high-orderharmonic spectra remain essentially unchanged at longer wavelengths: the two isotopic species are indistinguishable away from the structural minimum in the recombination matrix elements. In the vicinity of the structural minimum, $\mathrm{CD}_{4}$ shows much smaller suppression compared to the lighter isotopolog. As the result, we will neither show nor discuss the frozen-nuclei HHG spectra for longer wavelengths.

Returning to the $800-\mathrm{nm}, 1-\mathrm{PW} \mathrm{cm}{ }^{-2}$ case [Fig. 6(b)], the results obtained using different approaches for the treatment of subcycle nuclear motion remain similar within the harmonic plateau (below $\approx 150 \mathrm{eV}$ ), but start to differ closer to the structural minimum. As expected, the factorized AC approach faithfully reproduces the shape of the frozen-nuclei spectra $\left[\mathrm{CH}_{4}\right.$ : solid red line; $\mathrm{CD}_{4}$ : solid blue line, Fig. 6(b)], and yields a pronounced minimum around $194 \mathrm{eV}$. However, the minimum is completely filled in when using a more elaborate treatment (ND or DS), reflecting the spreading of the nuclear wave packet between ionization and recombination. The magnitude of the isotope effects is also much reduced, and is possibly inverted (see Sec. IV C below). Furthermore, laser-field modification of vibronic dynamics may begin to play a role.

Calculated HHG spectra for single-sided recollision at $1.2 \mu \mathrm{m}$ are collected in Fig. 7. At $300 \mathrm{TW} \mathrm{cm} \mathrm{cm}^{-2}$, the cutoff is found at $\approx 145 \mathrm{eV}$ (H140), well below the structural minimum. On the low-energy side of the plateau (below $\approx 80 \mathrm{eV}$ ), the three approaches to the treatment of nuclear motion (AC, ND, and DS) yield very similar results. This is not unexpected: this low-energy part of the spectrum corresponds to recollision time delays below $1.7 \mathrm{fs}$, which were explored by the 800 -nm results above. At longer time delays, correlated vibronic calculations (both ND and DS) now predict significantly higher HHG intensity than the direct-product AC approximation (but still two orders of magnitude lower than the frozen-nuclei results). The enhancement is due to the coordinate dependence of the strong-field ionization [Eq. (30)]. Close to the peak of the laser electric field, it leads to a substantial population of the nuclear basis functions excited along the $v_{3}$ (asymmetric stretch) and $v_{4}$ (asymmetric bend) normal modes. For $\mathrm{CH}_{4}$ and linear polarization along $X$ (Fig. 1), the initial amplitudes of the singly excited $v_{4}\left(v_{3}\right)$ on the dominant $D_{3}$ electronic surface reach $14 \%$ (33\%) of the vertical-ionization amplitude at $300 \mathrm{TW} \mathrm{cm} \mathrm{cm}^{-2}$, increasing to $14 \%(36 \%)$ at $1 \mathrm{PW} \mathrm{cm}-2$.
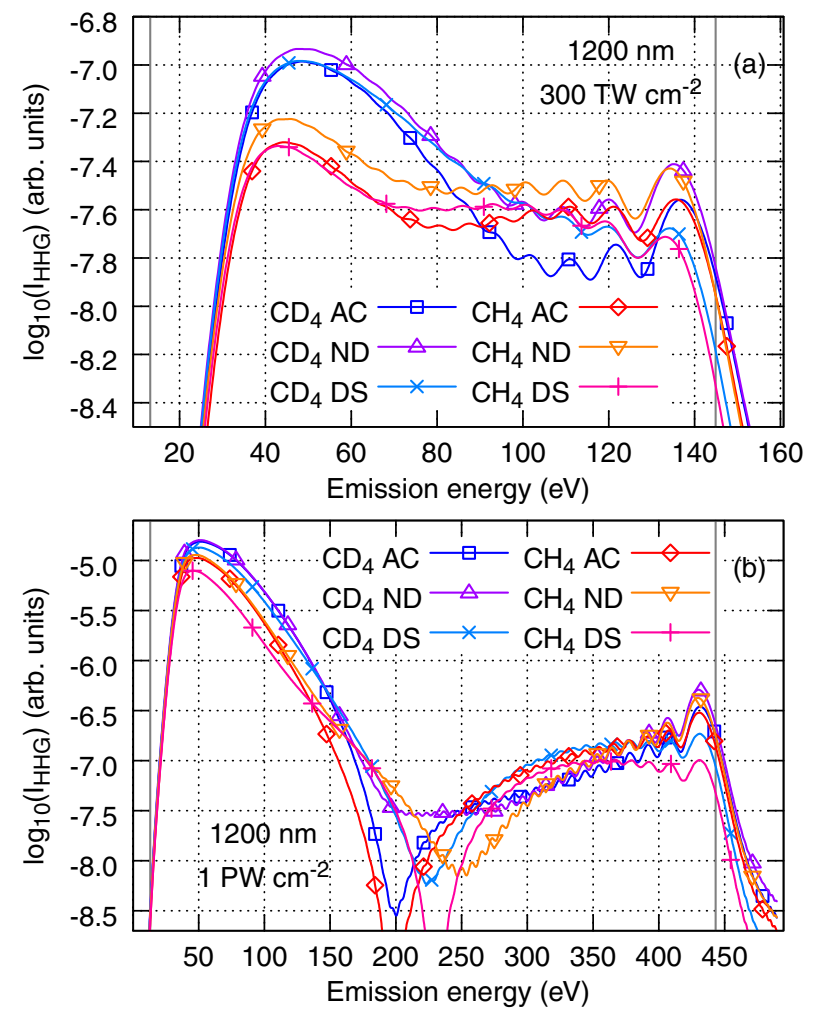

FIG. 7. MC-SFA-GWP high-order-harmonic spectrum of methane in $1.2-\mu \mathrm{m}$ driving field. (a) $300 \mathrm{TW} \mathrm{cm}^{-2}$. (b) $1 \mathrm{PW} \mathrm{cm}^{-2}$. Also see Fig. 6 caption. The HHG spectra for the frozen nuclear configuration are off the scale, and are not shown.

Ionization-induced nuclear wave-packet reshaping is smaller, but still substantial for $\mathrm{CD}_{4}: v_{4}\left(v_{3}\right)$ relative amplitudes of $9 \%(19 \%)$ at $300 \mathrm{TW} \mathrm{cm}-2$, increasing to $10 \%(21 \%)$ at 1 $\mathrm{PW} \mathrm{cm}^{-2}$. The initially excited component of the vibronic wave packet reaches the half revival faster (after $\approx 2 \mathrm{fs}$ delay), while the higher magnitude of the revival (Fig. 5) compensates for the reduced population relative to the vertical ionization. In the heavier $\mathrm{CD}_{4}$, the $v_{4} / \nu_{3}$ half revivals occur later (after $\approx 2.2 \mathrm{fs}$ ), so that the ND and AC spectra remain similar until much closer to the cutoff. Finally, the ND (no laser coupling in the vibronic dynamics) and DS (full laser coupling) spectra begin to diverge close to the cutoff, indicating that fieldinduced bound-state dynamics becomes important beyond 2 -fs delays.

At the higher $1 \mathrm{PW} \mathrm{cm}^{-2}$ intensity [Fig. 7(b)], the harmonics cutoff now extends to $443 \mathrm{eV}$ (H429). The structural minimum in the recombination matrix elements is now well within the plateau region, inducing a false cutoff near $200 \mathrm{eV}$. The direct product form (AC) remains a reasonable approximation early within the plateau (up to $\approx 1.5 \mathrm{fs} ; 160 \mathrm{eV}$ emission energy). At higher emission energies, both the correlations between the vibronic and continuum dynamics and the field-induced vibronic transitions become important. The position of the structural minimum is shifted to higher photon energies (225-250 eV, depending on the species and the details of the treatment). A similar shift of an HHG feature was experimentally observed [43] in PACER experiments on $\mathrm{H}_{2}$, where however coordinate dependence of the recombination matrix elements moves the minimum to lower energies. 

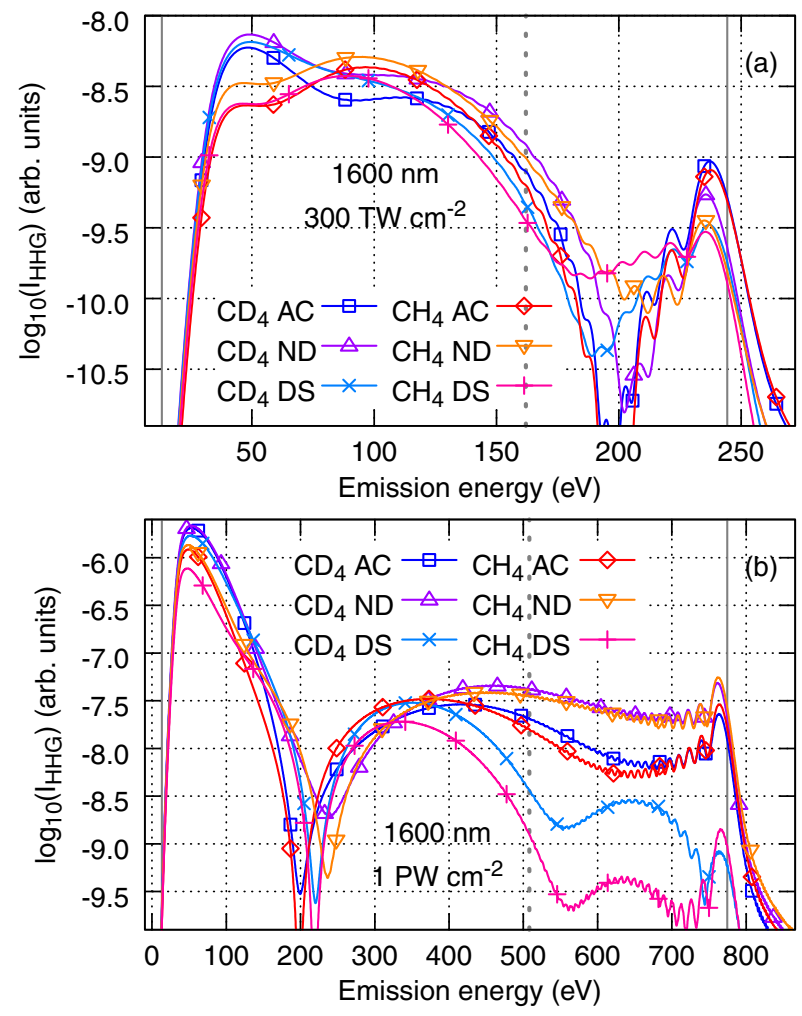

FIG. 8. MC-SFA-GWP high-order-harmonic spectrum of methane in 1.6- $\mu \mathrm{m}$ driving field. (a) $300 \mathrm{TW} \mathrm{cm}^{-2}$. (b) $1 \mathrm{PW} \mathrm{cm}^{-2}$. Also see Fig. 6 caption. The HHG spectra for the frozen nuclear configuration are off the scale, and are not shown. Parts of the ND and DS spectra between the dotted vertical grey line and the cutoff may show increased computational errors; see Sec. III D.

Vibronic dynamics "fills in" the structural minimum $\left(\mathrm{CD}_{4}\right.$ $\mathrm{ND}$ : purple line with triangles; $\mathrm{CH}_{4} \mathrm{ND}$ : orange line with inverted triangles). At the same time, field-induced vibronic transitions sharpen the minimum again $\left(\mathrm{CD}_{4} \mathrm{DS}\right.$ : teal line with crosses; $\mathrm{CH}_{4}$ DS: pink line with plus signs). The isotope effects in the high-energy part of the plateau thus reflect a number of dynamics (see Sec. IV C below).

Our final example uses a 1.6- $\mu \mathrm{m}$ driver (Fig. 8). Already at $300 \mathrm{TW} \mathrm{cm}^{-2}$ [Fig. 8(a)] the cutoff extends beyond the structural minimum, to $244 \mathrm{eV}$ (H315). The qualitative features of the calculated HHG spectra are similar to the $1.2-\mu \mathrm{m}$, 1-PW cm${ }^{-2}$ case above: the simplified product representation (AC) is adequate at low energies (short times); correlated vibronic dynamics and laser-induced vibronic coupling are increasingly important at higher energies, and especially in the vicinity of the structural minimum. At the $1-\mathrm{PW} \mathrm{cm}^{-2}$ intensity, the still higher-order-harmonic cutoff $(775 \mathrm{eV}, \mathrm{H} 1000)$, leads to a particularly clean demonstration of a transition from largely pure, field-free vibronic dynamics to fully correlated dynamics under strong-field control [Fig. 8(b)]. On the left side of the plateau (before $170 \mathrm{eV} / 1.6 \mathrm{fs}$ ), the spectra are grouped by the isotopic species, with all three treatments (AC, ND, and DS) yielding very similar results for either species.

On the right-hand side of the plateau (beyond $450 \mathrm{eV} / 2.3 \mathrm{fs}$ ), the HHG spectra are instead grouped by the treatment of bound-continuum correlations and laser field effects in vibronic dynamics. For example, at $600 \mathrm{eV}$, the product form $\left(\mathrm{CD}_{4} \mathrm{AC}\right.$, blue line with squares) underestimates the HHG intensity by a factor of $\approx 3 \times$ compared the field-free correlated treatment of the vibronic dynamics $\left(\mathrm{CD}_{4} \mathrm{ND}\right.$, purple line with triangles). At the same time, the full treatment including field-induced vibronic transitions $\left(\mathrm{CD}_{4} \mathrm{DS}\right.$, teal line with crosses) predicts $\mathrm{HHG}$ yield at this energy $\approx 2.3 \times$ below the AC treatment, and $\approx 8 \times$ below the field-free dynamics prediction. For nuclear dynamics simulations neglecting fieldinduced vibronic transitions, isotopic effects in this region do not exceed $1.5 \times$. However, the full treatment $\left(\mathrm{CH}_{4} \mathrm{DS}\right.$, purple line with plus signs) predicts a very strong suppression of harmonic emission beyond $500 \mathrm{eV}$, which is likely to appear as a false cutoff in experiment. Clearly, neglecting field-induced bound-state transitions in the cation is not a viable treatment in the high-intensity and multicycle limit [65,71].

Finally, in the transition region around the structural minimum, both the position and the depth of the structural minimum are highly sensitive to the isotopic species, treatment of nonadiabatic dynamics, and laser-induced bound-state dynamics. Again similar to the $1.2-\mu \mathrm{m}, 1-\mathrm{PW} \mathrm{cm} \mathrm{cm}^{-2}$ case, correlated vibronic dynamics shifts the apparent position of the structural minimum to higher energies and reduces its contrast, while inclusion of the laser-induced vibronic dynamics partially reverses the shift. In $\mathrm{CH}_{4}$, the minimum is found at $198 \mathrm{eV}(\mathrm{AC}), 236 \mathrm{eV}(\mathrm{ND})$, and $219 \mathrm{eV}(\mathrm{DS})$. In $\mathrm{CD}_{4}$, the minima are shifted slightly to higher photon energies: $200 \mathrm{eV}$ (AC), $239 \mathrm{eV}$ (ND), and $220 \mathrm{eV}$ (DS).

\section{Isotope effects}

Because PACER experiments are routinely interpreted in terms of ionization-recollision time delays, it is instructive to examine the isotopic ratios of the high-order-harmonic spectra in Figs. 6-8 in a similar way. In the absence of continuum resonances, there exists a one-to-one mapping between the short-trajectory HHG spectrum and the ionization-recollision time delay [9]. This relationship breaks down close to sharp features in photorecombination matrix elements (see Sec. IV B and Refs. $[102,103,105,140])$. Although the emission time in these cases can still be recovered through the time-frequency analysis [141], there appears to be no unambiguous way of reconstructing the ionization-recollision time delay close to resonances. We therefore choose the classical "simple man's" mapping [9] (SMM) for the ionization-recombination time delay, with the twin caveats: (a) this mapping is known to be inaccurate for short trajectories, especially for the $800-\mathrm{nm}$ driver [7,141]; and (b) the mapping should be treated as undefined close to structural minima in the harmonic spectrum. The advantage of the SMM is that the range of possible time delays depends only on the wavelength of the driving laser, so that different intensities can be compared directly. The underlying assumption of the PACER method is that the isotope effects represented in this form are intensity and wavelength independent.

Calculated isotope effects for the 800-nm driving field are shown in Fig. 9. The available experimental data [41] (black error bars) were obtained at the estimated intensity of $200 \mathrm{TW} \mathrm{cm}^{-2}$, and can be most directly compared to the numerical results of Fig. 9(a), calculated at a somewhat higher 


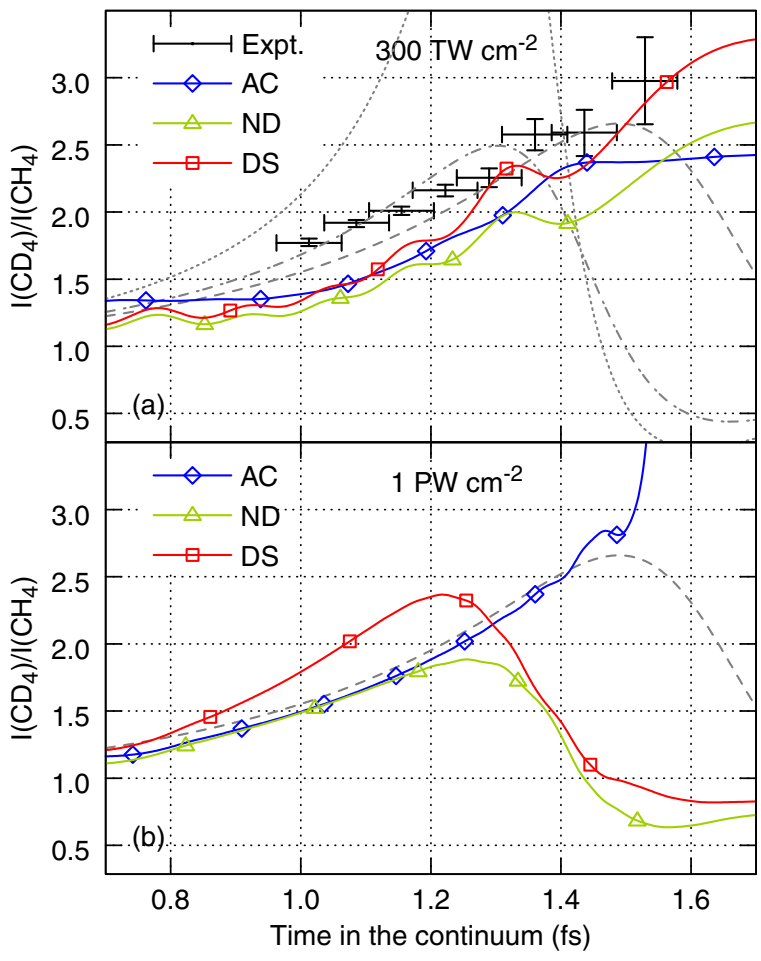

FIG. 9. Isotope effects in methane for the 800-nm driving field. Vertical axis: The ratio of the calculated HHG yields for $\mathrm{CD}_{4}$ and $\mathrm{CH}_{4}$ (Fig. 6). Horizontal axis: Time delay between ionization and recollision events, calculated from the harmonic energy using classical simple man's model (see text). AC (blue line with diamonds): direct product of the autocorrelation function and frozen-nuclei electron dynamics; ND (green line with triangles): correlated treatment, field-induced bound-state dynamics neglected; DS (red line with squares): the full treatment, including field-induced vibronic transitions. Dashed grey line gives the ratio of ground-state autocorrelation factors (Fig. 3). Dash-dotted and dotted grey lines in (a) represent the ratio of autocorrelation factors for $v_{3}$ and $v_{4}$ vibrationally excited initial wave packets (Fig. 5), respectively. Panels (a) and (b) correspond to laser intensity of 300 and $1000 \mathrm{TW} \mathrm{cm} \mathrm{cm}^{-2}$, respectively. Experimental points in (a) are from Ref. [41], measured at the estimated intensity of $200 \mathrm{TW} \mathrm{cm} \mathrm{cm}^{-2}$.

intensity of $300 \mathrm{TW} \mathrm{cm}^{-2}$. As expected from the similarity of the calculated spectra for the three approximations we consider here [Fig. 6(a)], the calculated PACER ratios are nearly identical. The simple ratio of the autocorrelation functions (Fig. 3) yields nearly identical results, except very close to the harmonic cutoff ( $t>1.5 \mathrm{fs})$. The agreement with the experiment is satisfactory, although the experimental isotope ratios are consistently slightly higher than the calculated values. A possible reason for the discrepancy is the vibrational excitation of the neutral molecule by the raising edge of the 8-fs pulse used in experiment [41], which is not included in the present single-cycle simulation. As can be seen from Fig. 5, population of the IR-active $v_{3}$ and $v_{4}$ vibrational modes in the initial wave packet is expected to increase isotope effects for the delays below 1.3 fs [dash-dotted and dotted lines in Fig. 9(a)].

Both the intrinsic correlations and laser-induced vibronic dynamics become important for the isotope effects at the

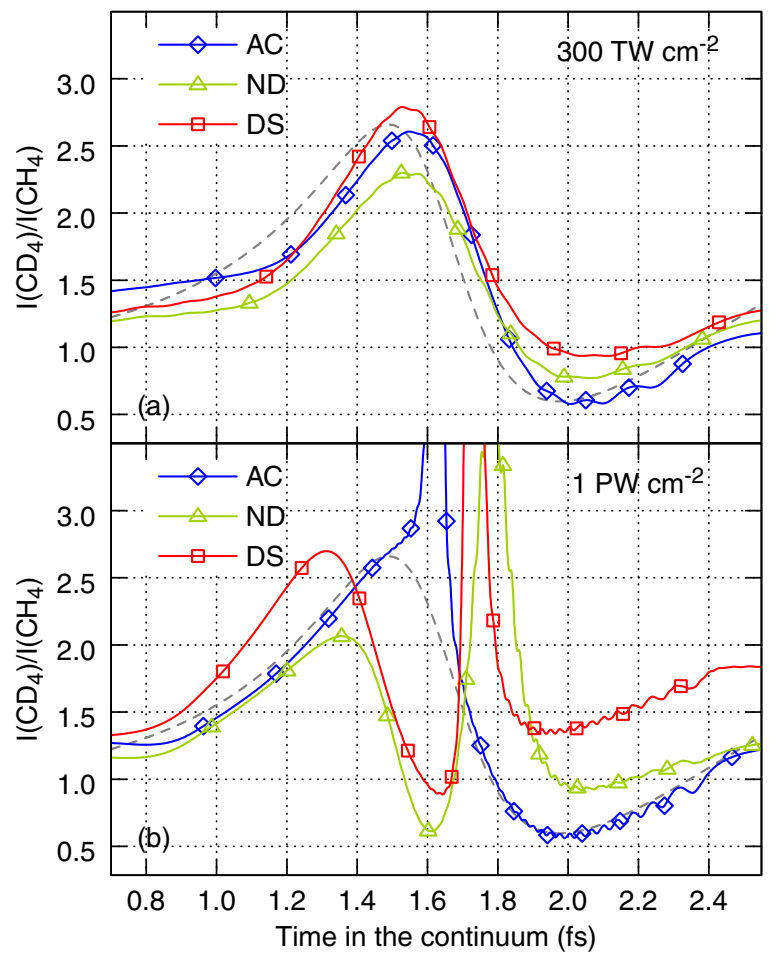

FIG. 10. Isotope effects in methane for $1.2-\mu \mathrm{m}$ driving field, from HHG data in Fig. 7. Also see Fig. 9 caption.

higher, $1 \mathrm{PW} \mathrm{cm}{ }^{-2}$ intensity [Fig. 9(b)]. Compared to the uncoupled approximation (AC, blue line with diamonds), correlations between the continuum and vibronic dynamics in the cation (ND, green line with triangles) substantially reduce the calculated isotope effects. Laser-induced vibronic dynamics (DS, red line with squares) partially counteracts this effect. Our best-effort calculation (DS) suggests that the observed isotope effects at this intensity should start decreasing beyond 1.2-fs delays, with inverse isotope effects predicted beyond 1.45 fs. We emphasize that the inverse isotope effect in this case is due to the structural minimum at $196 \mathrm{eV}$ in our photoionization matrix elements (Fig. 2) and the associated breakdown of the time-frequency mapping. An inverse isotope effect of a similar origin was previously predicted for the $\mathrm{D}_{2} / \mathrm{H}_{2}$ pair $[65,67]$. A change in the position of the photoionization resonance will also change the apparent time delay where the inverse isotope effect is predicted.

The simulated PACER results for the longer-wavelength, 1.2- $\mu \mathrm{m}$ driver and moderate $300-\mathrm{TW} \mathrm{cm}^{-2}$ intensity are shown in Fig. 10(a). Superficially, this PACER trace is remarkably similar to the DS (correlated and laser-coupled) result at $800 \mathrm{~nm}$ and $1 \mathrm{PW} \mathrm{cm}{ }^{-2}$ [Fig. 9(b)]: the isotope effect first increases, then changes sense at longer delay times. However, the physics behind the trace is entirely different. At $1.2 \mu \mathrm{m}$, the signal reflects the field-free vibronic dynamics in the transient cation, with neither vibronic-continuum correlations nor laser-driven vibronic dynamics qualitatively affecting the result. Thus, neglecting the laser coupling in the cation (ND) and neglecting both the laser coupling and vibronic-continuum correlations (AC) yield results very similar to the full simulation. All three simulated PACER traces are nearly on top of the simple ratio of the autocorrelation functions (dashed line). At 


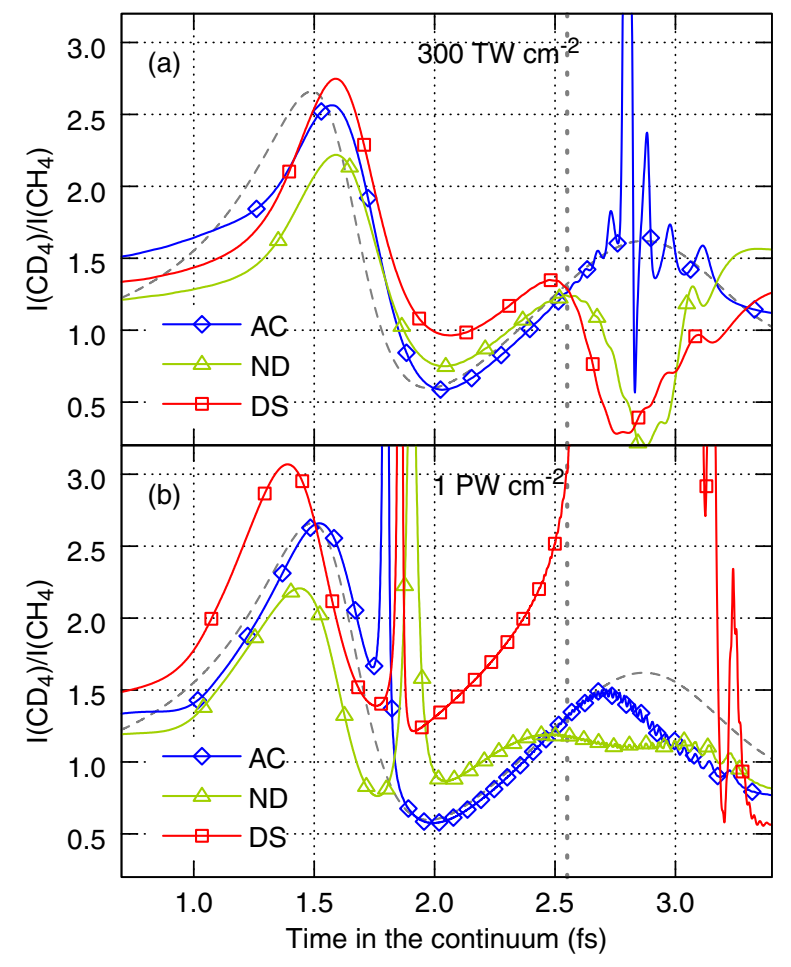

FIG. 11. Isotope effects in methane for $1.6-\mu \mathrm{m}$ driving field, from HHG data in Fig. 8. Time delays beyond the grey vertical dotted line at $2.6 \mathrm{fs}$ may suffer from increased numerical errors; see Sec. III D of the text. Also see Fig. 9 caption.

the $1.2 \mu \mathrm{m}$ wavelength, the laser cycle is long enough to allow vibronic wave packet in the cation to reach the half revival at the conical-intersection point (see Fig. 3 and Sec. IV A). The PACER trace containing a reversal of the isotope effect thus represents a true signature of the CI dynamics.

The situation becomes more complex at the higher $1 \mathrm{PW} \mathrm{cm}{ }^{-2}$ intensity [Fig. 10(b)]. Now, the harmonic spectrum extends far enough to access the structural minimum in the recombination matrix elements. As a result, the apparent isotope effects at time delays corresponding to the structural minimum (AC: $\approx 1.63 \mathrm{fs} ; \mathrm{ND}: \approx 1.78 \mathrm{fs} ; \mathrm{DS}: \approx 1.73 \mathrm{fs}$ ) become very large, and sensitive to the details of the treatment (AC: $14 \times$; ND: $3.9 \times$; DS: $175 \times)$. At time delays unaffected by the structural minimum $(t<1.55 \mathrm{fs}$ and $t>1.85 \mathrm{fs})$, the calculated PACER signal is qualitatively similar to the $300-\mathrm{TW} \mathrm{cm}^{-2}$ results. As was already seen above for the $800-\mathrm{nm}$ case, the influence of continuum-vibronic correlations and laser-driven vibronic dynamics increases at the higher intensity.

Finally, simulated PACER traces at $1.6 \mu \mathrm{m}$ wavelength are shown in Fig. 11. Already at the moderate $300-\mathrm{TW} \mathrm{cm}^{-2}$ intensity, harmonic cutoff reaches beyond the structural minimum. The corresponding PACER trace [Fig. 11(a)] is representative of the intrinsic vibronic dynamics for the delays below $\approx 2.5 \mathrm{fs}$. At longer times, the structural-minimum feature dominates. Further increasing the intensity [Fig. 11(b)] leads to a very complex spectrum, which loses nearly all information on the intrinsic vibronic dynamics beyond $\approx 1.7$ fs. If the intrinsic, field-free dynamics of the cation is of interest, extending the wavelength to $1.6 \mu \mathrm{m}$ therefore appears to offer little advantage.

\section{CONCLUSIONS AND OUTLOOK}

In this work, we introduced MC-SFA-GWP-a version of the molecular strong-field approximation which treats all electronic and nuclear degrees of freedom, including their correlations, quantum mechanically. The technique allows realistic simulations of the nuclear motion effects on high-orderharmonic emission in polyatomic molecules without invoking reduced-dimensionality models for the nuclear motion or the electronic structure.

We use the technique to model isotope effects in methane. The intermediate ${ }^{2} \mathrm{~F}_{2}$ electronic state of the $\mathrm{CH}_{4}{ }^{+}$cation transiently accessed by the HHG process possesses a symmetryrequired triple-state conical intersection at the Franck-Condon point. Simulations of the field-free vibronic dynamics of the ${ }^{2} F_{2}$ state in $\mathrm{CH}_{4}+$ indicates that a fraction of the initially prepared wave packet undergoes a half revival, accompanied by a sign change of the vibronic wave function, within $2.2 \mathrm{fs}$. This time scale is well within the laser-cycle duration of nearIR light, and is accessible with HHG spectroscopy. The revival is associated with population of an intermediate vibronic wave packet, composed of the singly excited $v_{4}\left(t_{2}\right)$ and $v_{2}(e)$ vibrational modes coupled to the degenerate components of the ${ }^{2} F_{2}$ electronic surface. In the space of nuclear coordinates, this wave packet forms a prolate spheroidal shell around the conical intersection. The revival times are determined predominantly by the strength of the vibronic coupling near the $\mathrm{CI}$, thus permitting its direct experimental measurement.

A well-understood difficulty in PACER and other HHG spectroscopies of molecules is the strong (Gaussian in time) suppression of the HHG emission due to the loss of nuclear wave-function overlap. We demonstrate that beyond $1.5 \mathrm{fs}$, nuclear motion in methane no longer causes a Gaussian suppression of the HHG signal, with the expected nuclear factor persisting at $\approx 1 \%$ level up to $3.5 \mathrm{fs}$-high enough to allow experimental detection. This unexpected persistence of the fraction of the HHG signal appears to be universal, and has been predicted for several other cations $[89,97]$.

We analyze and identify a number of physical mechanisms which contribute to the isotopic PACER signal in methane. At intensities below $300 \mathrm{TW} \mathrm{cm} \mathrm{cm}^{-2}$ and wavelengths below $1.2 \mu \mathrm{m}$, the autocorrelation contribution is dominant. This contribution derives from the intrinsic vibronic dynamics around the conical intersection. It manifests as an inverse isotope effect at $\approx 2.2 \mathrm{fs}$ ionization-recollision delay, and is wavelength and intensity independent. This effect has been predicted previously $[91,92]$. An experimental observation was claimed very recently [49].

The autocorrelation term is modified by a number of wavelength- and/or intensity-dependent contributions, including coordinate dependence of strong-field ionization amplitudes [64]; laser-driven vibronic dynamics in the neural and the transient cation [71]; the coordinate dependence of the photorecombination amplitudes $[43,65,67]$. For wavelengths beyond $\approx 1.6 \mu \mathrm{m}$ or intensities above $300 \mathrm{TW} \mathrm{cm} \mathrm{cm}^{-2}$, the latter contributions may dominate the PACER spectrum (see also Refs. [65,67]), even for a single-cycle driving pulse.

Furthermore, we demonstrate that the PACER concept breaks down for harmonic emission close to resonances (constructive or destructive) in the recombination matrix 
elements. At these energies, no simple relationship exists between the harmonic photon energy and the emission time. The isotopic ratio is then no longer representative of the intrinsic vibronic dynamics in the cation. For any given combination of the wavelength and intensity of the driving field, the PACER spectrogram resulting from a resonance may be indistinguishable from the signal due to the intrinsic vibronic dynamics. It is therefore essential to perform PACER experiments at a number of wavelengths and/or intensities. Features which appear at a fixed, or nearly fixed, emission energy are likely to originate from a resonance in the recombination matrix elements.

Although the present analysis focuses on one of the simplest polyatomic molecules, methane, both conical intersections and resonant features in photorecombination matrix elements are ubiquitous in polyatomic molecules. High-order-harmonic spectroscopy thus provides a tool for exploring both, in the regime not easily accessible with other techniques.

The effects discussed presently are of the subcycle nature, and are already present for a single-cycle driving pulse. For longer pulses, additional physical mechanisms will come into play $[64,68,71,77,79]$. A careful examination of these multicycle effects and their interaction with the intrinsic subcycle dynamics is one of the possible future applications of the MC-SFA-GWP approach.

\section{ACKNOWLEDGMENT}

S.P. acknowledges support from the U.S. DOD MURI Grant No. EP/N018680/1. M.S. acknowledges funding from the National Science and Engineering Research Council of Canada through the Discovery grant program.

\section{APPENDIX A: EVALUATION OF THE $d t_{1}$ INTEGRAL IN EQ. (26)}

We need to evaluate an integral in the form

$$
I=\int_{t_{0}}^{t} d t_{1} f\left(\vec{k}_{\mathrm{s}}\left(t_{1}\right)\right) e^{-i \phi_{d}\left(\vec{k}_{\mathrm{s}}\left(t_{1}\right), t, t_{1}\right)},
$$

where $\vec{k}_{\mathrm{S}}$ is in turn a function of $t_{1}$ [Eq. (27)] and $\phi_{d}$ is defined by Eq. (24). We assume that $f\left(\vec{k}_{\mathrm{s}}\right)$ depends at most linearly on $\vec{k}_{\mathrm{s}}$. Expanding $\phi_{d}$ through the third [98] order in $t_{1}$ around $t_{\mathrm{s}}$, changing integration variable to $\tau=t_{1}-t_{\mathrm{s}}$, and extending the integration limits to infinity, we obtain

$$
\begin{gathered}
I \approx \int_{-\infty}^{\infty} d \tau\left[f+\tau \frac{\partial f}{\partial \vec{k}_{\mathrm{s}}} \cdot \frac{\partial \vec{k}_{\mathrm{s}}}{\partial t_{\mathrm{s}}}+\tau \frac{\partial f}{\partial t_{\mathrm{s}}}\right] \\
\times e^{-i \phi_{d}-i\left(\partial \phi_{d} / \partial t_{\mathrm{s}}\right) \tau-(i / 2)\left(\partial^{2} \phi_{d} / \partial t_{\mathrm{s}}^{2}\right) \tau^{2}-(i / 6)\left(\partial^{3} \phi_{d} / \partial t_{\mathrm{s}}^{3}\right) \tau^{3}} \\
\frac{\partial^{3} \phi_{d}}{\partial t_{\mathrm{s}}^{3}}=-\frac{3 \hbar}{m} \frac{\vec{k}_{\mathrm{s}}^{2}}{\left(t-t_{\mathrm{s}}\right)^{2}}+\frac{3 e}{m} \frac{1}{t-t_{\mathrm{s}}} \vec{k}_{\mathrm{s}} \cdot \vec{F} \\
+\frac{e}{m} \vec{k}_{\mathrm{s}} \cdot \frac{\partial}{\partial t_{\mathrm{s}}} \vec{F}-\frac{e^{2}}{m \hbar} \vec{F}^{2}
\end{gathered}
$$

$$
\begin{gathered}
\frac{\partial \vec{k}_{\mathrm{s}}}{\partial t_{\mathrm{s}}}=\frac{\vec{k}_{\mathrm{s}}}{t-t_{\mathrm{s}}}-\frac{e}{\hbar} \vec{F}, \\
\frac{\partial \phi_{d}}{\partial t_{\mathrm{s}}}=-\frac{\hbar}{2 m} \vec{k}_{\mathrm{s}}^{2}-\frac{1}{\hbar} I_{\mathrm{p}}, \\
\frac{\partial^{2} \phi_{d}}{\partial t_{\mathrm{s}}^{2}}=-\frac{\hbar}{m} \frac{\vec{k}_{\mathrm{s}}^{2}}{t-t_{\mathrm{s}}}+e \vec{k}_{\mathrm{s}} \cdot \vec{F} .
\end{gathered}
$$

In Eq. (A2), $f, \phi_{d}$, their derivatives, and $\vec{F}$ are evaluated at $\vec{k}_{\mathrm{s}}$ and $t_{\mathrm{s}}$ pairs solving Eq. (28). For linearly polarized driving field, $\frac{\partial^{2} \phi_{d}}{\partial t_{\mathrm{s}}^{2}}$ [Eq. (A6)] and all but the last term in $\frac{\partial^{3} \phi_{d}}{\partial t_{\mathrm{s}}^{3}}$ [Eq. (A3)] vanish. For low-frequency fields, these terms remain negligible for moderate nonzero ellipticities as well (highorder-harmonic signal vanishes for large ellipticities [98]), so that

$$
\begin{gathered}
\frac{\partial^{2} \phi_{d}}{\partial t_{\mathrm{s}}^{2}} \approx 0, \\
\frac{\partial^{3} \phi_{d}}{\partial t_{\mathrm{s}}^{3}} \approx-\frac{e^{2}}{m \hbar} \vec{F}^{2} .
\end{gathered}
$$

We now note that [Eq. (10.4.32) of Ref. [142]]

$$
\begin{array}{r}
\int_{-\infty}^{\infty} e^{i a t^{3}+i x t} d t=\frac{2 \pi}{(3 a)^{1 / 3}} \operatorname{Ai}\left(\frac{x}{(3 a)^{1 / 3}}\right), \\
\int_{-\infty}^{\infty} t e^{i a t^{3}+i x t} d t=-i \frac{2 \pi}{(3 a)^{2 / 3}} \operatorname{Ai}^{\prime}\left(\frac{x}{(3 a)^{1 / 3}}\right),
\end{array}
$$

where Ai is an Airy function. [Equation (A8) is obtained by differentiating Eq. (A7) with respect to $x$.] Therefore, Eq. (A2) becomes

$$
\begin{gathered}
I \approx e^{-i \phi_{d}} 2 \pi\left[f\left(\frac{2 m \hbar}{e^{2} \vec{F}^{2}}\right)^{1 / 3} \operatorname{Ai}(\zeta)-i\left(\frac{\partial f}{\partial t_{\mathrm{s}}}+\frac{\partial f}{\partial \vec{k}_{\mathrm{s}}} \cdot \frac{\partial \vec{k}_{\mathrm{s}}}{\partial t_{\mathrm{s}}}\right)\right. \\
\left.\times\left(\frac{2 m \hbar}{e^{2} \vec{F}^{2}}\right)^{2 / 3} \mathrm{Ai}^{\prime}(\zeta)\right] \\
\zeta=\left(\frac{2 m}{e^{2} \hbar^{2} \vec{F}^{2}}\right)^{1 / 3}\left(I_{\mathrm{p}}+\frac{\hbar^{2} \vec{k}_{\mathrm{s}}^{2}}{2 m}\right)
\end{gathered}
$$

where again all quantities are evaluated at pairs $\vec{k}_{\mathrm{s}}, t_{\mathrm{s}}$ satisfying Eq. (28). If multiple roots are present, summation over all roots is implied.

\section{APPENDIX B: DERIVATION OF EQ. (30)}

The presence of the vibronic state energy expectations $E_{b \mathbf{m}}$ and $E_{a^{\prime \prime \prime} \mathbf{n}^{\prime \prime}}$, rather than the manifold average $I_{\mathrm{p}}$, in Eq. (31) is intuitively appealing, but requires some additional justification. Application of Eq. (A9) to the integral of Eq. (26) leads to

$$
\begin{aligned}
\Upsilon_{b \mathbf{m} a^{\prime \prime \prime} \mathbf{n}^{\prime \prime \prime}}= & \vec{F}\left(t_{\mathrm{s}}\right) \cdot \vec{R}_{b \mathbf{m} a^{\prime \prime \prime} \mathbf{n}^{\prime \prime \prime}}\left(\vec{k}_{\mathrm{s}}\right) 2 \pi\left(\frac{2 m}{e^{2} \hbar^{2} \vec{F}^{2}\left(t_{\mathrm{s}}\right)}\right)^{1 / 3} \operatorname{Ai}(\zeta) \\
& -2 i \pi \vec{F}\left(t_{\mathrm{s}}\right) \cdot \vec{R}_{b \mathbf{m} a^{\prime \prime \prime} \mathbf{n}^{\prime \prime \prime}}\left(\vec{k}_{\mathrm{s}}\right) \frac{\partial}{\partial t_{s}} \ln C_{a^{\prime \prime \prime} \mathbf{n}^{\prime \prime \prime}}\left(t_{\mathrm{s}}\right)\left(\frac{2 m}{e^{2} \hbar^{2} \vec{F}^{2}\left(t_{\mathrm{s}}\right)}\right)^{2 / 3} \operatorname{Ai}^{\prime}(\zeta)
\end{aligned}
$$




$$
\begin{aligned}
& -2 i \pi \vec{F}\left(t_{\mathrm{s}}\right) \cdot \vec{R}_{b \mathbf{m} a^{\prime \prime \prime} \mathbf{n}^{\prime \prime \prime}}\left(\vec{k}_{\mathrm{s}}\right) \frac{\partial}{\partial t_{s}} \ln D_{b^{\prime} \mathbf{m}^{\prime} b \mathbf{m}}\left(t, t_{\mathrm{s}}\right)\left(\frac{2 m}{e^{2} \hbar^{2} \vec{F}^{2}\left(t_{\mathrm{s}}\right)}\right)^{2 / 3} \mathrm{Ai}^{\prime}(\zeta) \\
& -2 i \pi \frac{\partial}{\partial \vec{k}_{\mathrm{s}}}\left[\vec{F}\left(t_{\mathrm{s}}\right) \cdot \vec{R}_{b \mathbf{m} a^{\prime \prime \prime} \mathbf{n}^{\prime \prime \prime}}\left(\vec{k}_{\mathrm{s}}\right)\right] \cdot\left(\frac{\hbar \vec{k}_{\mathrm{s}}}{t-t_{\mathrm{s}}}-e \vec{F}\left(t_{\mathrm{s}}\right)\right)\left(\frac{2 m}{e^{2} \hbar^{2} \vec{F}^{2}\left(t_{\mathrm{s}}\right)}\right)^{2 / 3} \mathrm{Ai}^{\prime}(\zeta),
\end{aligned}
$$

where we have neglected the $t_{\mathrm{s}}$ and $\vec{k}_{\mathrm{s}}$ dependence of the recombination dipole and the overall prefactor and $\zeta$ is given by Eq. (A10). Formal differentiation of Eqs. (22) and (23) gives

$$
\begin{aligned}
\frac{\partial}{\partial t_{1}} D_{b^{\prime} \mathbf{m}^{\prime} b \mathbf{m}}\left(t, t_{1}\right)= & \frac{i}{\hbar} e^{i E_{\mathrm{I}}\left(t-t_{1}\right) / \hbar}\left\langle\mathbf{m}^{\prime}\right|\left\langle X_{b^{\prime}}\right| \hat{U}_{\mathrm{I}}\left(t, t_{1}\right) \\
& \times\left[\hat{H}_{\mathrm{I}}\left(t_{1}\right)-E_{\mathrm{I}}\right]\left|X_{b}\right\rangle|\mathbf{m}\rangle, \\
\frac{\partial}{\partial t_{1}} C_{a \mathbf{n} a^{\prime} \mathbf{n}^{\prime}}\left(t_{1}, t_{0}\right)= & -\frac{i}{\hbar} e^{i E_{\mathrm{N}}\left(t_{1}-t_{0}\right) / \hbar}\langle\mathbf{n}|\left\langle\Phi_{a}\right|\left[\hat{H}_{0}\left(t_{1}\right)-E_{\mathrm{N}}\right] \\
& \times \hat{U}_{0}\left(t_{1}, t_{0}\right)\left|\Phi_{a^{\prime}}\right\rangle\left|\mathbf{n}^{\prime}\right\rangle .
\end{aligned}
$$

If the Hamiltonians $\hat{H}_{\mathrm{I}}$ and $\hat{H}_{0}$ are diagonally dominant in the basis of corresponding vibronic product states, Eqs. (B2) and (B3) reduce to

$$
\begin{aligned}
\frac{\partial}{\partial t_{1}} D_{b^{\prime} \mathbf{m}^{\prime} b \mathbf{m}}\left(t, t_{1}\right) & \approx \frac{i}{\hbar}\left(E_{b \mathbf{m}}-E_{\mathrm{I}}\right) D_{b^{\prime} \mathbf{m}^{\prime} b \mathbf{m}}\left(t, t_{1}\right), \\
\frac{\partial}{\partial t_{1}} C_{a \mathbf{n} a^{\prime} \mathbf{n}^{\prime}}\left(t_{1}, t_{0}\right) & \approx-\frac{i}{\hbar}\left(E_{a \mathbf{n}}-E_{\mathrm{N}}\right) C_{a \mathbf{n} a^{\prime} \mathbf{n}^{\prime}}\left(t_{1}, t_{0}\right),
\end{aligned}
$$

where $E_{b \mathbf{m}}$ and $E_{a \mathbf{n}}$ are given by Eqs. (32) and (33).

Substituting Eqs. (B4) and (B5) into Eq. (B1), we note that the first three terms are in fact the lowest-order contributions to the Taylor expansion of the first term in Eq. (30) for $\delta E=$ $\left(E_{b \mathbf{m}}-E_{a^{\prime \prime \prime} \mathbf{n}^{\prime \prime \prime}}\right)-I_{\mathrm{p}}$. Similar expansion for the second term corresponds to a term quadratic in $\tau$, neglected in deriving Eq. (A9). To within the accuracy expected from Eq. (30), we can therefore replace $I_{\mathrm{p}}$ by $\left(E_{b \mathbf{m}}-E_{a^{\prime \prime \prime} \mathbf{n}^{\prime \prime \prime}}\right)$ in the last term of Eq. (B1) as well, giving Eq. (31). The exponential part of Eq. (31) coincides with the result of the weak-field asymptotic tunneling theory (WFAT) [143].

\section{APPENDIX C: EVALUATION OF THE FOURIER-TRANSFORM INTEGRALS IN Eq. (18)}

Evaluation of matrix elements appearing in Eq. (18) requires calculation of Fourier transforms of the "cradle" orbitals $\vec{\phi}_{b a}^{\mathrm{C}}$ and products of the Dyson orbitals $\phi_{b a}^{\mathrm{D}}$ and a dipole operator $\vec{r}$. Here, both Dyson and cradle orbitals are given by an expansion over atom-centered Cartesian Gaussiantype orbitals. The desired integrals are readily obtained by a simple modification of standard one-electron integral packages.

Indeed, closely following the approach of Ahlrichs [144], the primitive integral $I_{\mathbf{0 , 0}}$ is given by [cf.
Eq. (7) of [144]]

$$
\begin{gathered}
I_{\mathbf{0}, \mathbf{0}}=\left\langle\mathbf{0}\left|e^{i \vec{k} \cdot \vec{r}}\right| \mathbf{0}\right\rangle \\
=\int d \vec{r} e^{-\alpha|\vec{r}-\mathbf{A}|^{2}} e^{i \vec{k} \cdot \vec{r}} e^{-\beta|\vec{r}-\mathbf{B}|^{2}} \\
=e^{\xi|\mathbf{B}-\mathbf{A}|^{2}}\left(\frac{\pi}{\zeta}\right)^{3 / 2} e^{i \vec{k} \cdot \mathbf{P}} e^{-\vec{k}^{2} / 4 \zeta}, \\
|\mathbf{a}\rangle=\left(x-A_{x}\right)^{a_{x}}\left(y-A_{y}\right)^{a_{y}}\left(z-A_{z}\right)^{a_{z}} e^{-\alpha|\vec{r}-\mathbf{A}|^{2}}, \\
\mathbf{P}=\frac{\alpha \mathbf{A}+\beta \mathbf{B}}{\alpha+\beta}, \\
\zeta=\alpha+\beta, \\
\xi=\frac{\alpha \beta}{\alpha+\beta} .
\end{gathered}
$$

Functions $|\mathbf{a}\rangle$ are unnormalized Cartesian Gaussians with quantum numbers $\mathbf{a}$ and exponent $\alpha$, centered at $\mathbf{A}$ [Eq. (1) of [144]], and analogously for $|\mathbf{b}\rangle$. Applying the usual generating operators [M of Eqs. (17) and (29) of [144]], we immediately obtain the recursion relation

$$
I_{\mathbf{a}+\mathbf{1}_{p}, \mathbf{b}}=\left(\mathbf{P}_{p}-\mathbf{A}_{p}+\frac{i k_{p}}{2 \zeta}\right) I_{\mathbf{a}, \mathbf{b}}+\frac{a_{p}}{2 \zeta} I_{\mathbf{a}-\mathbf{1}_{p}, \mathbf{b}}+\frac{b_{p}}{2 \zeta} I_{\mathbf{a}, \mathbf{b}-\mathbf{1}_{p}},
$$

where $p$ is a Cartesian direction $(p=x, y, z), \mathbf{a}=\left(a_{x}, a_{y}, a_{z}\right)$, and $\mathbf{1}_{p}$ is a unit three-vector containing 1 in position $p$. Noting that

$$
r_{p}|\mathbf{a}\rangle=\left|\mathbf{a}+\mathbf{1}_{p}\right\rangle+A_{p}|\mathbf{a}\rangle,
$$

we then obtain

$$
\begin{aligned}
\left\langle\mathbf{a}\left|r_{p} e^{i \vec{k} \cdot \vec{r}}\right| \mathbf{b}\right\rangle & =I_{\mathbf{a}+\mathbf{1}_{p}, \mathbf{b}}+A_{p} I_{\mathbf{a}, \mathbf{b}} \\
& =\left(\mathbf{P}_{p}+\frac{i k_{p}}{2 \zeta}\right) I_{\mathbf{a}, \mathbf{b}}+\frac{a_{p}}{2 \zeta} I_{\mathbf{a}-\mathbf{1}_{p}, \mathbf{b}}+\frac{b_{p}}{2 \zeta} I_{\mathbf{a}, \mathbf{b}-\mathbf{1}_{p}} .
\end{aligned}
$$

We note that Eqs. (C6) and (C8) are nearly identical to the standard recursion relations for the overlap and dipole integrals $[145,146]$. Finally, by choosing $\mathbf{b}=\mathbf{0}, \beta=0$, and $\mathbf{B}=\mathbf{0}$ in Eqs. (C6) and (C8), we obtain the desired expressions for the Fourier transforms of the primitive Gaussian $|\mathbf{a}\rangle$ and its first Cartesian moments.
[1] E. Goulielmakis, V. S. Yakovlev, A. L. Cavalieri, M. Uiberacker, V. Pervak, A. Apolonski, R. Kienberger, U. Kleineberg, and F. Krausz, Science 317, 769 (2007).
[2] F. Krausz and M. Ivanov, Rev. Mod. Phys. 81, 163 (2009).

[3] P. Salières, A. Maquet, S. Haessler, J. Caillat, and R. Taïeb, Rep. Prog. Phys. 75, 062401 (2012). 
[4] F. Lepine, M. Y. Ivanov, and M. J. J. Vrakking, Nat. Photon. 8, 195 (2014).

[5] F. Calegari, G. Sansone, S. Stagira, C. Vozzi, and M. Nisoli, J. Phys. B 49, 062001 (2016).

[6] K. Ramasesha, S. R. Leone, and D. M. Neumark, in Annual Review of Physical Chemistry, edited by M. A. Johnson and T. J. Martinez (Annual Reviews, Palo Alto, CA, 2016), Vol. 67, pp. 41-63.

[7] S. Haessler, J. Caillat, and P. Salières, J. Phys. B 44, 203001 (2011).

[8] M. Ferray, A. L'Huillier, X. F. Li, L. A. Lompre, G. Mainfray, and C. Manus, J. Phys. B 21, L31 (1988).

[9] P. B. Corkum, Phys. Rev. Lett. 71, 1994 (1993).

[10] T. Kanai, E. J. Takahashi, Y. Nabekawa, and K. Midorikawa, Phys. Rev. A 77, 041402 (2008).

[11] A. Fleischer, O. Kfir, T. Diskin, P. Sidorenko, and O. Cohen, Nat. Photon. 8, 543 (2014).

[12] J. Itatani, J. Levesque, D. Zeidler, H. Niikura, H. Pépin, J. C. Kieffer, P. B. Corkum, and D. M. Villeneuve, Nature (London) 432, 867 (2004).

[13] S. Haessler, J. Caillat, W. Boutu, C. Giovanetti-Teixeira, T. Ruchon, T. Auguste, Z. Diveki, P. Breger, A. Maquet, B. Carré et al., Nat. Phys. 6, 200 (2010).

[14] W. Schwarz, Angew. Chem. Int. Ed. 45, 1508 (2006).

[15] J. P. Farrell, S. Petretti, J. Förster, B. K. McFarland, L. S. Spector, Y. V. Vanne, P. Decleva, P. H. Bucksbaum, A. Saenz, and M. Gühr, Phys. Rev. Lett. 107, 083001 (2011).

[16] A. Ferré, A. E. Boguslavskiy, M. Dagan, V. Blanchet, B. D. Bruner, F. Burgy, A. Camper, D. Descamps, B. Fabre, N. Fedorov et al., Nat. Commun. 6, 5952 (2015).

[17] O. Smirnova, Y. Mairesse, S. Patchkovskii, N. Dudovich, D. Villeneuve, P. Corkum, and M. Y. Ivanov, Nature (London) 460, 972 (2009).

[18] O. Smirnova, S. Patchkovskii, Y. Mairesse, N. Dudovich, D. Villeneuve, P. Corkum, and M. Y. Ivanov, Phys. Rev. Lett. 102, 063601 (2009).

[19] Y. Mairesse, J. Higuet, N. Dudovich, D. Shafir, B. Fabre, E. Mével, E. Constant, S. Patchkovskii, Z. Walters, M. Y. Ivanov et al., Phys. Rev. Lett. 104, 213601 (2010).

[20] R. Torres, T. Siegel, L. Brugnera, I. Procino, J. G. Underwood, C. Altucci, R. Velotta, E. Springate, C. Froud, I. C. E. Turcu et al., Phys. Rev. A 81, 051802 (2010).

[21] Z. Diveki, A. Camper, S. Haessler, T. Auguste, T. Ruchon, B. Carré, P. Salières, R. Guichard, J. Caillat, A. Maquet et al., New J. Phys. 14, 023062 (2012).

[22] J. Leeuwenburgh, B. Cooper, V. Averbukh, J. P. Marangos, and M. Ivanov, Phys. Rev. Lett. 111, 123002 (2013).

[23] K. T. Kim, C. Zhang, T. Ruchon, J.-F. Hergott, T. Auguste, D. M. Villeneuve, P. B. Corkum, and F. Quéré, Nat. Photon. 7, 651 (2013).

[24] P. M. Kraus, B. Mignolet, D. Baykusheva, A. Rupenyan, L. Horný, E. F. Penka, G. Grassi, O. I. Tolstikhin, J. Schneider, F. Jensen et al., Science 350, 790 (2015).

[25] B. D. Bruner, Z. Mašín, M. Negro, F. Morales, D. Brambila, M. Devetta, D. Faccialà, A. G. Harvey, M. Ivanov, Y. Mairesse et al., Faraday Discuss. 194, 369 (2016).

[26] N. L. Wagner, A. Wüest, I. P. Christov, T. Popmintchev, X. Zhou, M. M. Murnane, and H. C. Kapteyn, Proc. Natl. Acad. Sci. USA 103, 13279 (2006).
[27] Y. Mairesse, D. Zeidler, N. Dudovich, M. Spanner, J. Levesque, D. M. Villeneuve, and P. B. Corkum, Phys. Rev. Lett. 100, 143903 (2008).

[28] L. S. Spector, M. Artamonov, S. Miyabe, T. Martinez, T. Seideman, M. Guehr, and P. H. Bucksbaum, Nat. Commun. 5, 3190 (2014).

[29] W. Li, X. Zhou, R. Lock, S. Patchkovskii, A. Stolow, H. C. Kapteyn, and M. M. Murnane, Science 322, 1207 (2008).

[30] A. D. Shiner, B. E. Schmidt, C. Trallero-Herrero, H. J. Wörner, S. Patchkovskii, P. B. Corkum, J.-C. Kieffer, F. Légaré, and D. M. Villeneuve, Nat. Phys. 7, 464 (2011).

[31] R. Cireasa, A. E. Boguslavskiy, B. Pons, M. C. H. Wong, D. Descamps, S. Petit, H. Ruf, N. Thiré, A. Ferré, J. Suarez et al., Nat. Phys. 11, 654 (2015).

[32] O. Smirnova, Y. Mairesse, and S. Patchkovskii, J. Phys. B 48, 234005 (2015).

[33] D. Shafir, H. Soifer, B. D. Bruner, M. Dagan, Y. Mairesse, S. Patchkovskii, M. Y. Ivanov, O. Smirnova, and N. Dudovich, Nature (London) 485, 343 (2012).

[34] X. Zhou, R. Lock, W. Li, N. Wagner, M. M. Murnane, and H. C. Kapteyn, Phys. Rev. Lett. 100, 073902 (2008).

[35] H. J. Wörner, H. Niikura, J. B. Bertrand, P. B. Corkum, and D. M. Villeneuve, Phys. Rev. Lett. 102, 103901 (2009).

[36] G. Orenstein, O. Pedatzur, A. J. Uzan, B. D. Bruner, Y. Mairesse, and N. Dudovich, Phys. Rev. A 95, 051401 (2017).

[37] W. Boutu, S. Haessler, H. Merdji, P. Breger, G. Waters, M. Stankiewicz, L. J. Frasinski, R. Taïeb, J. Caillat, A. Maquet et al., Nat. Phys. 4, 545 (2008).

[38] X. Zhou, R. Lock, N. Wagner, W. Li, H. C. Kapteyn, and M. M. Murnane, Phys. Rev. Lett. 102, 073902 (2009).

[39] M. Lewenstein, P. Balcou, M. Y. Ivanov, A. L'Huillier, and P. B. Corkum, Phys. Rev. A 49, 2117 (1994).

[40] M. Lein, Phys. Rev. Lett. 94, 053004 (2005).

[41] S. Baker, J. S. Robinson, C. A. Haworth, H. Teng, R. A. Smith, C. C. Chirilă, M. Lein, J. W. G. Tisch, and J. P. Marangos, Science 312, 424 (2006).

[42] J. P. Marangos, S. Baker, N. Kajumba, J. S. Robinson, J. W. G. Tisch, and R. Torres, Phys. Chem. Chem. Phys. 10, 35 (2008).

[43] S. Baker, J. S. Robinson, M. Lein, C. C. Chirilă, R. Torres, H. C. Bandulet, D. Comtois, J. C. Kieffer, D. M. Villeneuve, J. W. G. Tisch et al., Phys. Rev. Lett. 101, 053901 (2008).

[44] T. Kanai, E. J. Takahashi, Y. Nabekawa, and K. Midorikawa, New J. Phys. 10, 025036 (2008).

[45] S. Haessler, W. Boutu, M. Stankiewicz, L. J. Frasinski, S. Weber, J. Caillat, R. Taïeb, A. Maquet, P. Breger, P. Monchicourt et al., J. Phys. B 42, 134002 (2009).

[46] H. Mizutani, S. Minemoto, Y. Oguchi, and H. Sakai, J. Phys. B 44, 081002 (2011).

[47] P. M. Kraus and H. J. Wörner, Chem. Phys. Chem. 14, 1445 (2013).

[48] A. Zaïr, T. Siegel, S. Sukiasyan, F. Risoud, L. Brugnera, C. Hutchison, Z. Diveki, T. Auguste, J. W. Tisch, P. Salières et al., Chem. Phys. 414, 184 (2013).

[49] P. Lan, M. Ruhmann, L. He, C. Zhai, F. Wang, X. Zhu, Q. Zhang, Y. Zhou, M. Li, M. Lein et al., Phys. Rev. Lett. 119, 033201 (2017).

[50] S. Baker, J. S. Robinson, C. A. Haworth, C. C. Chirilă, M. Lein, J. W. G. Tisch, and J. P. Marangos, J. Mod. Opt. 54, 1011 (2007).

[51] M. Lein, J. Phys. B 40, R135 (2007). 
[52] S. Patchkovskii, Phys. Rev. Lett. 102, 253602 (2009).

[53] R. A. Ganeev, L. B. Elouga Bom, J. Abdul-Hadi, M. C. H. Wong, J. P. Brichta, V. R. Bhardwaj, and T. Ozaki, Phys. Rev. Lett. 102, 013903 (2009).

[54] M. C. H. Wong, J. P. Brichta, and V. R. Bhardwaj, Phys. Rev. A 81, 061402 (2010).

[55] R. Torres, T. Siegel, L. Brugnera, I. Procino, J. G. Underwood, C. Altucci, R. Velotta, E. Springate, C. Froud, I. C. E. Turcu et al., Opt. Express 18, 3174 (2010).

[56] C. Vozzi, R. Torres, M. Negro, L. Brugnera, T. Siegel, C. Altucci, R. Velotta, F. Frassetto, L. Poletto, P. Villoresi et al., Appl. Phys. Lett. 97, 241103 (2010).

[57] M. C. H. Wong, J.-P. Brichta, M. Spanner, S. Patchkovskii, and V. R. Bhardwaj, Phys. Rev. A 84, 051403 (2011).

[58] M. C. H. Wong, A. T. Le, A. F. Alharbi, A. E. Boguslavskiy, R. R. Lucchese, J. P. Brichta, C. D. Lin, and V. R. Bhardwaj, Phys. Rev. Lett. 110, 033006 (2013).

[59] C. Hutchison, R. A. Ganeev, M. Castillejo, I. Lopez-Quintas, A. Zaïr, S. J. Weber, F. McGrath, Z. Abdelrahman, M. Oppermann, M. Martin et al., Phys. Chem. Chem. Phys. 15, 12308 (2013).

[60] A. F. Alharbi, A. E. Boguslavskiy, N. Thiré, B. E. Schmidt, F. Légaré, T. Brabec, M. Spanner, and V. R. Bhardwaj, Phys. Rev. A 92, 041801 (2015).

[61] J. P. Marangos, J. Phys. B 49, 132001 (2016).

[62] D. R. Yarkony, in Conical Intersections: Electronic Structure, Dynamics and Spectroscopy, edited by W. Domcke, D. R. Yarkony, and H. Köppel (World Scientific, Singapore, 2004), Chap. 2, pp. 41-127.

[63] D. R. Yarkony, Chem. Rev. 112, 481 (2012).

[64] S. Chelkowski, A. Conjusteau, T. Zuo, and A. D. Bandrauk, Phys. Rev. A 54, 3235 (1996).

[65] C. C. Chirilă and M. Lein, Phys. Rev. A 77, 043403 (2008).

[66] A. D. Bandrauk, S. Chelkowski, and H. Lu, J. Phys. B 42, 075602 (2009).

[67] C. C. Chirilă and M. Lein, Chem. Phys. 366, 54 (2009).

[68] R. Daniele, G. Castiglia, P. P. Corso, E. Fiordilino, F. Morales, and G. Orlando, J. Mod. Opt. 56, 751 (2009).

[69] A. D. Bandrauk, S. Chelkowski, S. Kawai, and H. Lu, Phys. Rev. Lett. 101, 153901 (2008).

[70] J. Zhao and Z. Zhao, Phys. Rev. A 78, 053414 (2008).

[71] G. Castiglia, P. P. Corso, R. Daniele, E. Fiordilino, B. Frusteri, and F. Morales, Laser Phys. 23, 095301 (2013).

[72] T. Bredtmann, S. Chelkowski, and A. D. Bandrauk, J. Phys. Chem. A 116, 11398 (2012).

[73] Y. Zheng, Z. Zeng, R. Li, and Z. Xu, Phys. Rev. A 85, 023410 (2012).

[74] N.-T. Nguyen, V.-H. Hoang, and V.-H. Le, Phys. Rev. A 88, 023824 (2013).

[75] H. Ahmadi, A. Maghari, H. Sabzyan, A. R. Niknam, and M. Vafaee, Phys. Rev. A 90, 043411 (2014).

[76] X.-B. Bian and A. D. Bandrauk, Phys. Rev. Lett. 113, 193901 (2014).

[77] F. Morales, P. Rivière, M. Richter, A. Gubaydullin, M. Ivanov, O. Smirnova, and F. Martín, J. Phys. B 47, 204015 (2014).

[78] H. Ahmadi, M. Vafaee, and A. Maghari, Phys. Rev. A 94, 033415 (2016).

[79] M. Lara-Astiaso, R. E. F. Silva, A. Gubaydullin, P. Rivière, C. Meier, and F. Martín, Phys. Rev. Lett. 117, 093003 (2016).

[80] M.-Z. Li, G.-R. Jia, and X.-B. Bian, J. Chem. Phys. 146, 084305 (2017).
[81] X.-Y. Miao and H.-N. Du, Phys. Rev. A 87, 053403 (2013).

[82] Z. B. Walters, S. Tonzani, and C. H. Greene, J. Phys. B 40, F277 (2007).

[83] Z. B. Walters, S. Tonzani, and C. H. Greene, Chem. Phys. 366, 103 (2009).

[84] F. H. M. Faisal, Theor. Chem. Acc. 127, 175 (2010).

[85] M. Falge, V. Engel, and M. Lein, Phys. Rev. A 81, 023412 (2010).

[86] C. B. Madsen, M. Abu-samha, and L. B. Madsen, Phys. Rev. A 81, 043413 (2010).

[87] P. G. Lisinetskaya and R. Mitrić, Phys. Rev. A 83, 033408 (2011).

[88] A.-T. Le, T. Morishita, R. R. Lucchese, and C. D. Lin, Phys. Rev. Lett. 109, 203004 (2012).

[89] J. Förster and A. Saenz, Chem. Phys. Chem. 14, 1438 (2013).

[90] S. Patchkovskii and M. S. Schuurman, J. Phys. Chem. A 118, 12069 (2014).

[91] T. Mondal and A. J. C. Varandas, J. Chem. Theory Comput. 10, 3606 (2014).

[92] T. Mondal and A. J. C. Varandas, J. Chem. Phys. 143, 014304 (2015).

[93] B. Jayachander Rao and A. J. C. Varandas, Phys. Chem. Chem. Phys. 17, 6545 (2015).

[94] B. Jayachander Rao and A. J. C. Varandas, J. Phys. Chem. A 119, 12367 (2015).

[95] B. Jayachander Rao and A. J. C. Varandas, J. Phys. Chem. A 119, 4856 (2015).

[96] M. Vacher, L. Steinberg, A. J. Jenkins, M. J. Bearpark, and M. A. Robb, Phys. Rev. A 92, 040502 (2015).

[97] C. Arnold, O. Vendrell, and R. Santra, Phys. Rev. A 95, 033425 (2017).

[98] M. Y. Ivanov, T. Brabec, and N. Burnett, Phys. Rev. A 54, 742 (1996).

[99] A. Becker and F. Faisal, J. Phys. B 38, R1 (2005).

[100] S. V. Popruzhenko, J. Phys. B 47, 204001 (2014).

[101] O. Smirnova, M. Spanner, and M. Ivanov, J. Mod. Opt. 54, 1019 (2007).

[102] O. Smirnova and M. Ivanov, arXiv:1304.2413.

[103] O. Smirnova and M. Ivanov, in Attosecond and XUV Physics: Ultrafast Dynamics and Spectroscopy, edited by T. Schultz and M. Vrakking (Wiley-VCH, Weinheim, 2014), Chap. 7, pp. 201-256.

[104] M. Y. Amusia and N. A. Cherepkov, Case Stud. At. Phys. 5, 47 (1975).

[105] S. Patchkovskii, O. Smirnova, and M. Spanner, J. Phys. B 45, 131002 (2012).

[106] M. Spanner and S. Patchkovskii, Phys. Rev. A 80, 063411 (2009).

[107] M. Spanner and S. Patchkovskii, Chem. Phys. 414, 10 (2013).

[108] R. L. Martin and D. A. Shirley, J. Chem. Phys. 64, 3685 (1976).

[109] S. Patchkovskii, Z. Zhao, T. Brabec, and D. M. Villeneuve, Phys. Rev. Lett. 97, 123003 (2006).

[110] S. Patchkovskii, Z. Zhao, T. Brabec, and D. M. Villeneuve, J. Chem. Phys. 126, 114306 (2007).

[111] T. H. Dunning, J. Chem. Phys. 90, 1007 (1989).

[112] R. A. Kendall, T. H. Dunning Jr., and R. J. Harrison, J. Chem. Phys. 96, 6796 (1992).

[113] G. Herzberg, Molecular Spectra and Molecular Structure. III. Electronic Spectra and Electronic Structure of Polyatomic Molecules (D. Van Nostrand, Princeton, NJ, 1966). 
[114] M. Jacox, J. Phys. Chem. Ref. Data 32, 1 (2003).

[115] S. Lias, in NIST Chemistry WebBook, NIST Standard Reference Database Number 69, edited by P. Linstrom and W. Mallard (National Institute of Standards and Technology, Gaithersburg MD, 2017), retrieved September 4, 2017.

[116] J. Berkowitz, Atomic and Molecular Photoabsorption. Absolute Partial Cross Sections (Academic Press, Amsterdam, 2015).

[117] S. R. Langhoff and E. R. Davidson, Int. J. Quantum Chem. 8, 61 (1974).

[118] K. Kimura, S. Katsumata, Y. Achiba, T. Yamazaki, and S. Iwata, Handbook of HeI Photoelectron Spectra of Fundamental Organic Molecules (Japan Scientific Societies Press, Tokyo, 1981).

[119] M. S. Schuurman and D. R. Yarkony, J. Chem. Phys. 127, 094104 (2007).

[120] B. N. Papas, M. S. Schuurman, and D. R. Yarkony, J. Chem. Phys. 129, 124104 (2008).

[121] J. Dillon, D. R. Yarkony, and M. S. Schuurman, J. Chem. Phys. 134, 044101 (2011).

[122] F. Gadéa and M. Pélissier, J. Chem. Phys. 93, 545 (1990).

[123] I. D. Petsalakis, G. Theodorakopoulos, C. A. Nicolaides, and R. J. Buenker, Chem. Phys. Lett. 185, 359 (1991).

[124] T. Pacher, H. Köppel, and L. Cederbaum, J. Chem. Phys. 95, 6668 (1991).

[125] A. Gołębiewski, Faraday Trans. 57, 1849 (1961).

[126] J. N. Murrell, J. Chem. Phys. 32, 767 (1960).

[127] L. S. Cederbaum, J. Schirmer, and H.-D. Meyer, J. Phys. A 22, 2427 (1989).

[128] P.-O. Löwdin, Phys. Rev. 97, 1474 (1955).

[129] L. C. Lee, E. Phillips, and D. L. Judge, J. Chem. Phys. 67, 1237 (1977).

[130] J. A. R. Samson, G. N. Haddad, T. Masuoka, P. N. Pareek, and D. A. L. Kilcoyne, J. Chem. Phys. 90, 6925 (1989).
[131] J. W. Au, G. Cooper, G. R. Burton, and C. E. Brion, Chem. Phys. 187, 305 (1994).

[132] J. W. Au, G. Cooper, G. R. Burton, T. N. Olney, and C. E. Brion, Chem. Phys. 178, 615 (1993).

[133] K. Kameta, N. Kouchi, M. Ukai, and Y. Hatano, J. Electron. Spectrosc. Relat. Phenom. 123, 225 (2002).

[134] E. A. Reinsch, J. Chem. Phys. 83, 5784 (1985).

[135] I. Cacelli, V. Carravetta, R. Moccia, and A. Rizzo, J. Phys. Chem. 92, 979 (1988).

[136] B. N. Cabral Tenorio, M. A. Chaer Nascimento, S. Coriani, and A. B. Rocha, J. Chem. Theory Comput. 12, 4440 (2016).

[137] W. H. Press, S. A. Teukolsky, W. T. Vetterling, and B. P. Flannery, Numerical Recipes in Fortran 77: The Art of Scientific Computing, 2nd ed. (Cambridge University Press, Cambridge, England, 2003).

[138] D. J. Tannor, Introduction to Quantum Mechanics: A Timedependent Perspective (University Science Books, Sausalito, 2007).

[139] E. Goll, G. Wunner, and A. Saenz, Phys. Rev. Lett. 97, 103003 (2006).

[140] O. Smirnova, S. Patchkovskii, Y. Mairesse, N. Dudovich, and M. Y. Ivanov, Proc. Nat. Acad. Sci. USA 106, 16556 (2009).

[141] C. C. Chirilă, I. Dreissigacker, E. V. van der Zwan, and M. Lein, Phys. Rev. A 81, 033412 (2010).

[142] M. Abramowitz and I. A. Stegun, Handbook of Mathematical Functions: With Formulas, Graphs, and Mathematical Tables, 10th ed. (Dover, New York, 1972).

[143] O. I. Tolstikhin and T. Morishita, Phys. Rev. A 95, 033410 (2017).

[144] R. Ahlrichs, Phys. Chem. Chem. Phys. 8, 3072 (2006).

[145] S. Obara and A. Saika, J. Chem. Phys. 84, 3963 (1986).

[146] S. Obara and A. Saika, J. Chem. Phys. 89, 1540 (1988). 